\title{
EFFECT OF CARBOHYDRATE-PROTEIN SUPPLEMENTATION ON RESISTANCE EXERCISE PERFORMANCE, PERCEIVED EXERTION, AND SALIVARY CORTISOL
}

By

Michael J. Welikonich

B.S., East Stroudsburg University, 1997

M.S., Bloomsburg University, 2004

\author{
Submitted to the Graduate Faculty of \\ The School of Education in partial fulfillment \\ of the requirements for the degree of \\ Doctor of Philosophy
}

University of Pittsburgh 


\title{
UNIVERSITY OF PITTSBURGH
}

\section{SCHOOL OF EDUCATION}

This dissertation was presented

By

\author{
Michael J. Welikonich
}

It was defended on

October 14, 2010

and approved by

Fredric L. Goss, Associate Professor, School of Education

Robert J. Robertson, Professor, School of Education

Kim Crawford, Assistant Professor, Health and Rehabilitation Sciences

Elizabeth F. Nagle, Assistant Professor, School of Education 
Copyright (C) by Michael J. Welikonich

2010 


\section{EFFECT OF CARBOHYDRATE-PROTEIN SUPPLEMENTATION ON RESISTANCE EXERCISE PERFORMANCE, PERCEIVED EXERTION, AND SALIVARY CORTISOL}

Michael J. Welikonich, PhD

University of Pittsburgh, 2010

Carbohydrate-protein (CHO-PRO) and carbohydrate (CHO) beverages are two popular nutritional supplements currently used by many athletes to improve exercise performance. Research regarding these supplements effectiveness during resistance exercise (RE) has been inconsistent. PURPOSE: The purpose of this investigation was to determine the effect of CHO-PRO versus CHO supplementation and a placebo (PLA) on RE performance, perceived exertion during RE, and post-exercise salivary cortisol. METHODS: Twenty seven college aged (18 to 30 yrs) healthy males who participated in recreational strength training were recruited from the university community to participate in this investigation. Subjects randomly received either a: 1) CHO-PRO; 2) CHO; or 3) PLA supplement 15 minutes prior to exercise, between every other set during exercise, and immediately post-exercise. Subjects completed a RE session consisting of multiple sets of seated leg press exercise starting with 1 set of 10 repetitions at 40,50 , and $60 \%$ of 1 repetition maximum (RM), continuing with multiple sets of 8 to 10 repetitions at $70 \%$ of their $1 \mathrm{RM}$ until fatigue. Ratings of perceived exertion for the active muscle group (RPE-AM) and overall body (RPE-O) were measured immediately following each set and Session-RPE was measured 30 minutes after the RE session concluded. Salivary cortisol samples were collected approximately 15 minutes prior to 
exercise and 30 minutes post-exercise. RESULTS: $\mathrm{CHO}-\mathrm{PRO}$ and $\mathrm{CHO}$ supplementation resulted in a significantly greater total number of repetitions performed to fatigue than a PLA. CHO supplementation resulted in an attenuated RPE-AM response at $25 \%$ and $50 \%$ of total volume of work performed compared to the CHO-PRO supplementation or a PLA. There were no significant differences for RPE-O or SessionRPE between the three treatment groups. Finally, CHO supplementation resulted in an attenuation of post-exercise salivary cortisol response compared to CHO-PRO supplementation or a PLA. CONCLUSION: The results of this investigation suggest that both CHO-PRO and CHO supplementation before and during RE can have a positive impact on exercise performance which may ultimately lead to enhanced muscular adaptations and improved sport performance. 


\section{TABLE OF CONTENTS}

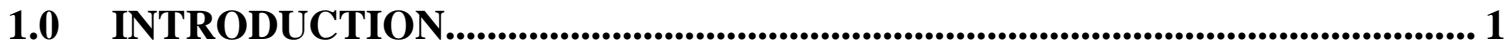

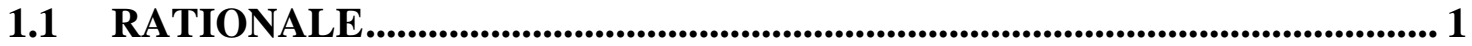

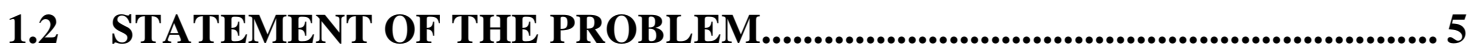

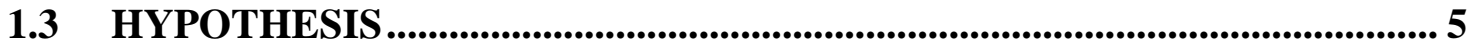

1.4 SIGNIFICANCE ................................................................................................ 6

2.0 LITERATURE REVIEW ........................................................................................ 7

2.1 ACUTE RESPONSES TO RESISTANCE EXERCISE.................................. 7

2.1.1 Physiological.................................................................................................... 7

2.1.1.1 Blood Glucose/Muscle Glycogen................................................................. 7

2.1.1.2 Hormonal Responses .................................................................................... 9

2.1.1.2.1 Cortisol ...................................................................................................... 9

2.1.1.2.2 Salivary Cortisol Measures .................................................................. 10

2.1.1.2.3 Salivary Cortisol and Resistance Exercise ...................................... 10

2.1.1.3 Muscle Protein Synthesis/Degradation ................................................... 12

2.1.1.4 Central and Peripheral Fatigue ............................................................... 15

2.1.2 Perceptual............................................................................................... 18

2.1.2.1 Ratings of Perceived Exertion (RPE) ...................................................... 18

2.1.2.2 Physiological Mediators .......................................................................... 19

2.1.2.3 RPE during Resistance Exercise ............................................................. 21

2.1.2.4 Session RPE ........................................................................................... 23 
2.2 CARBOHYDRATE SUPPLEMENTATION ................................................. 25

2.2.1 Possible Mechanisms for Acute Responses ................................................... 25

2.2.2 Effect on Aerobic Exercise............................................................................. 26

2.2.3 Effect on Resistance Exercise ................................................................. 28

2.2.4 Effect on Perceived Exertion ............................................................................. 31

2.3 CARBOHYDRATE-PROTEIN SUPPLEMENTATION .............................. 32

2.3.1 Possible Mechanisms for Acute Responses ................................................... 33

2.3.2 Effect on Aerobic Exercise........................................................................... 34

2.3.3 Effect on Resistance Exercise .......................................................................... 36

2.3.4 Effect on Perceived Exertion ............................................................................ 38

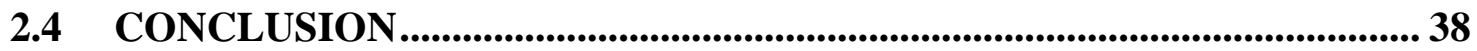

3.0 METHODS …............................................................................................................ 41

3.1 EXPERIMENTAL DESIGN ............................................................................... 41

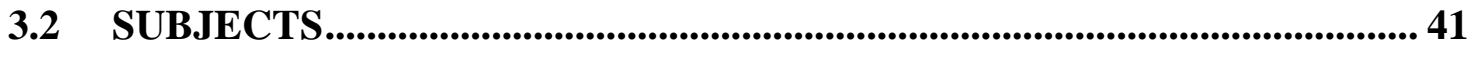

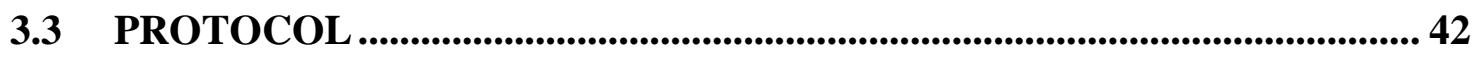

3.3.1 Protocol Overview ............................................................................................... 42

3.3.2 Day 1 .............................................................................................................. 43

3.3.2.1 Height, Weight, Body Composition............................................................ 44

3.3.2.2 Orientation to OMNI-RES......................................................................... 44

3.3.2.3 1 Repetition Maximum ................................................................................. 45

3.3.2.4 Diet Record ........................................................................................... 46

3.3.3 Day 2 .............................................................................................................. 47

3.3.3.1 Supplementation Protocol ........................................................................... 47 
3.3.3.2 Resistance Exercise Protocol................................................................... 48

3.3.4 Measurement of Salivary Cortisol .............................................................. 50

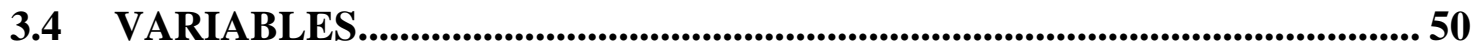

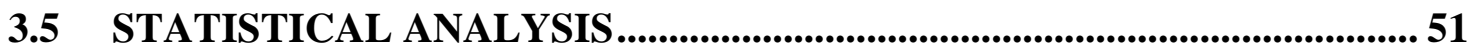

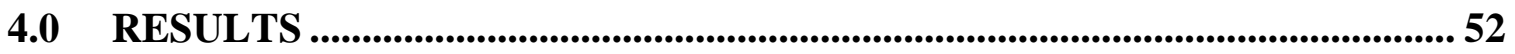

4.1 SUBJECT CHARACTERISTICS ................................................................ 52

4.2 RESISTANCE EXERCISE PERFORMANCE ............................................... 55

4.3 PERCEIVED EXERTION ................................................................................ 58

4.4 SALIVARY CORTISOL ...................................................................................... 61

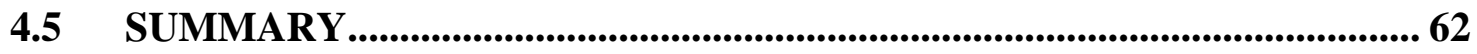

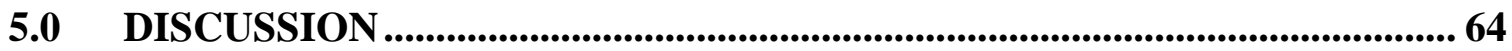

5.1 RESISTANCE EXERCISE PERFORMANCE ................................................ 65

5.2 PERCEIVED EXERTION ................................................................................... 69

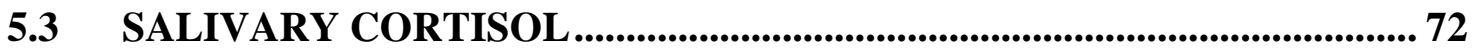

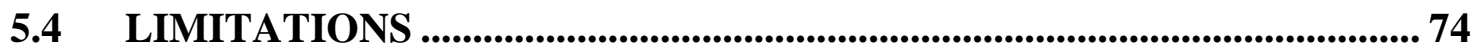

$5.5 \quad$ RECOMMENDATIONS FOR FUTURE RESEARCH................................ 77

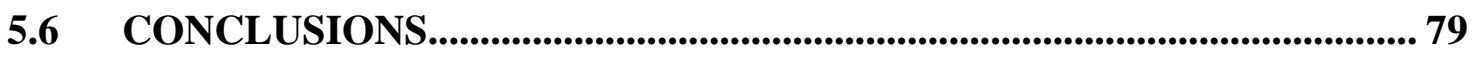

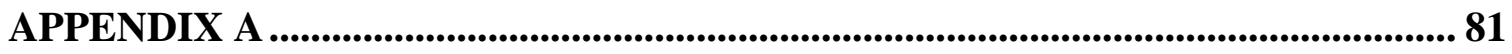

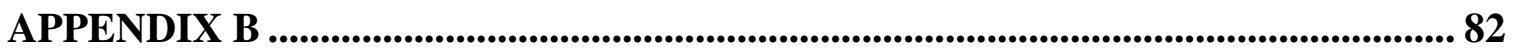

APPENDIX C ................................................................................................................ 83

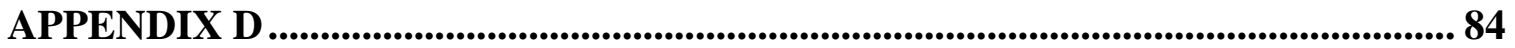

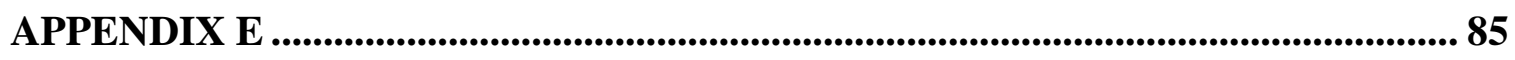

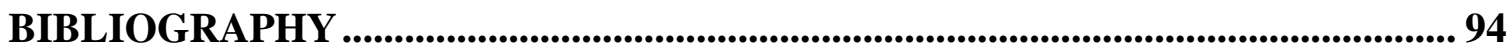




\section{LIST OF TABLES}

Table 1: A theory to explain the cause of central fatigue during exercise

(Blomstrand, 2001)..................................................................................................................... 18

Table 2: Summary of CHO-PRO supplementation and resistance exercise.............. 40

Table 3: Supplementation Beverage Formulation .............................................................. 48

Table 4: Subject Descriptive Characteristics. ....................................................................... 53

Table 5: Dietary Energy Intake and Macronutrient Composition. .............................. 54

Table 6: Total Supplement Intake Before and During RE Session............................... 55 


\section{LIST OF FIGURES}

Figure 1: Salivary cortisol response to various intensity resistance exercise protocols (Crewther et al., 2008).

Figure 2: OMNI perceived exertion scale for resistance exercise (OMNI-RES). ..... 23

Figure 3: OMNI perceived exertion scale for resistance exercise (OMNI-RES). .... 45

Figure 4: Resistance exercise protocol and measurement time points. 49

Figure 5: Total Repetitions Performed Following Supplementation and Training

Protocol.

Figure 6: Total Volume of Work Performed Following Supplementation and

Training Protocol

Figure 7: Relative Volume of Work (LBM) Following Supplementation and

Training Protocol.

Figure 8: RPE-AM at \% of Total Volume of Work Performed. 59

Figure 9: RPE-O at \% of Total Volume of Work Performed................................... 59

Figure 10: Session RPE-AM .................................................................................................... 60

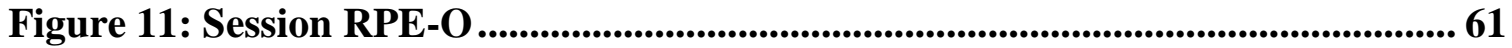

Figure 12: Salivary Cortisol Responses ........................................................... 62 


\subsection{INTRODUCTION}

\subsection{RATIONALE}

Within recent years, nutritional supplementation has been adopted by athletes, trainers, and coaches who seek safe and legal methods to improve exercise performance. Carbohydrate-protein (CHO-PRO) and carbohydrate (CHO) beverages are two popular nutritional supplements currently consumed by amateur and professional athletes. Ingesting a $\mathrm{CHO}$ and electrolyte beverage during exercise provides an immediate fuel source for working muscles and helps to offset body fluid losses from sweating to prevent dehydration (Tsintzas and Williams, 1998). Previous research has shown CHO supplementation to be effective for improving performance, attenuating perceived exertion, and reducing post-exercise cortisol levels during aerobic endurance exercise (Utter et al., 2007, Yaspelkis et al., 1993; Wright et al., 1991; Neufer et al., 1987).

Current evidence suggests that CHO-PRO supplementation during aerobic endurance exercise can have similar or greater benefits on performance when compared to CHO supplementation alone (Saunders et al., 2004; Ivy et al., 2003; Romano et al., 2006). Researchers have also shown that CHO-PRO supplementation can result in improved muscular adaptations, favorable hormonal responses, and enhanced recovery following resistance exercise (RE) compared to CHO supplementation (Baty et al., 2007; 
Bird et al., 2006; Rasmussen et al., 2001). Yet, it remains unclear whether CHO-PRO supplementation can improve performance, attenuate perceived exertion, or reduce postexercise salivary cortisol responses to RE. This is of interest because attenuation of perceived exertion may allow an athlete to perform a greater volume of work due to reduced feelings of discomfort and fatigue. Furthermore, the reduction of post-exercise cortisol may improve the balance of muscle protein synthesis/degradation and aid in recovery.

The phosphagen system (ATP-PC) has traditionally been considered a primary source of energy during resistance training, with a portion supplied by glycolysis. Recently, researchers have demonstrated that muscle glycogen stores can significantly decrease by approximately 20 to $40 \%$ during moderate intensity, high volume RE ranging from 36 to 50 total repetitions performed at 35 to $70 \%$ of maximal strength and lasting approximately 55 minutes or longer (Haff et al., 2000; MacDougall et al., 1999; Pascoe et al., 1993; Robergs et al., 1991; Tesch et al., 1996, 1998). This decrease in muscle glycogen may contribute to increased muscular weakness, decreased maximal muscular strength, and decreased force production due to a limited ability of glycogen to provide energy (Yaskpelkis et al., 1993; Jacobs et al., 1981; Hepburn et al., 1982).

Additionally, evidence suggests that carbohydrate availability is a mediator of perceived exertion during endurance exercise (Noble and Robertson, 1996; Utter et al., 2007). As muscle glycogen and blood glucose levels decrease, resulting muscular fatigue requires the recruitment of additional muscle fibers and causes an increased outflow in the motor cortex, which in turn can increase perceived exertion (Utter et al., 2007). By increasing carbohydrate availability through supplementation, it is anticipated that muscle 
glycogen stores would be maintained, and as a result, attenuate perceived exertion and improve performance during RE.

Evidence regarding the addition of protein to a carbohydrate supplement during exercise has been inconsistent. A few studies (Baty et al., 2007; Romano et al., 2006) have shown no improvement in performance from CHO-PRO compared to $\mathrm{CHO}$ supplementation, while other studies (Saunders et al., 2004; Ivy et al., 2003) have shown improvements in performance from CHO-PRO supplementation. Investigators have demonstrated that the addition of protein to a carbohydrate supplement during exercise may have additional benefits not observed in CHO supplementation alone. It is believed that the plasma insulin response increases in an effort to reduce significant decreases in muscle glycogen that occur during high volume RE (Ivy et al., 2003; Van Loon et al., 2000; Zawadzki et al., 1992). Additionally, evidence has shown that branched-chain amino acids (leucine, isoleucine, and valine) found in most protein supplements, can attenuate perceived exertion and reduce mental fatigue during exercise by influencing the synthesis, concentration, and release of neurotransmitters in the brain (Blomstrand et al., 1997). As a result, the use of a CHO-PRO supplement may improve performance and attenuate perceived exertion during RE to a greater extent when compared to a CHO only supplement.

Moderate intensity, high volume RE has been shown to elicit acute anabolic and catabolic hormonal responses in the body. Acute increases in anabolic hormones such as insulin and testosterone function to enhance protein synthesis in skeletal muscle and promote muscle growth. Conversely, increases in catabolic hormones such as cortisol attempt to provide energy in response to declines in muscle glycogen and blood glucose 
by stimulating gluconeogenesis and peripheral release of substrates. Cortisol stimulates protein degradation and decreases protein synthesis in muscle cells resulting in increased amino acid availability in the circulation to be converted to energy via gluconeogenesis (Haff et al., 2003; Utter et al., 2007).

Investigators have shown that performing RE without supplementation will result in a significant post-exercise increase in salivary cortisol indicative of increased muscle protein breakdown (Crewther et al., 2008; McGuigan et al., 2004). While a few studies have shown a reduced post-exercise plasma cortisol response to RE due to CHO-PRO supplementation (Baty et al., 2007; Bird et al., 2006; Williams et al., 2002), it appears that the post-exercise salivary cortisol response to RE due to CHO-PRO supplementation has not been examined.

Additionally, cortisol is a physiological marker for stress and some studies suggest it may be a mediator of perceived exertion during exercise (Utter et al., 2007). Cortisol typically increases significantly during the later portion of prolonged exercise and is correlated with higher ratings of perceived exertion. Utter et al. (1999) found that higher levels of plasma glucose due to carbohydrate supplementation were associated with lower levels of cortisol and attenuated ratings of perceived exertion during prolonged cycling. Therefore, it is also of interest to examine the effects of CHO-PRO and $\mathrm{CHO}$ supplementation on the post-exercise salivary cortisol response to RE. 


\subsection{STATEMENT OF THE PROBLEM}

The purpose of this investigation was to determine the effect of carbohydrate-protein (CHO-PRO) versus carbohydrate (CHO) supplementation and a placebo (PLA) on RE

performance in a sample of male college aged recreational athletes. A secondary purpose was to determine the effect of CHO-PRO versus CHO supplementation and PLA on perceived exertion during RE, and post-exercise salivary cortisol in a sample of college aged recreational athletes.

\subsection{HYPOTHESIS}

1. It was hypothesized that CHO-PRO supplementation will increase performance, reflected by volume of work output (sets $\times$ repetitions $\times$ weight), during $\mathrm{RE}$ when compared to CHO supplementation or the PLA condition.

2. It was hypothesized that CHO-PRO supplementation will result in a comparatively greater attenuation of perceived exertion for the active muscle (RPE-AM) and overall body (RPE-O) during RE compared to $\mathrm{CHO}$ supplementation or the PLA condition.

3. It was hypothesized that CHO-PRO supplementation will result in a comparatively greater attenuation of perceived exertion for the entire exercise 
session (Session RPE) when compared to CHO supplementation or the PLA condition.

4. It was hypothesized that CHO-PRO supplementation will result in a reduced preexercise to post-exercise salivary cortisol response when compared to $\mathrm{CHO}$ supplementation or the PLA condition.

\subsection{SIGNIFICANCE}

It is anticipated that the findings from this investigation will inform athletes, trainers, and coaches regarding the use of $\mathrm{CHO}-\mathrm{PRO}$ and $\mathrm{CHO}$ supplementation during RE. Athletes will benefit if CHO-PRO or CHO supplementation can improve the volume of work performed during a RE session, resulting in increased muscular adaptations. If CHOPRO or CHO supplementation is found to attenuate perceived exertion during resistance training, then athletes can adopt this technique to lessen the intensity of effort, stress, and discomfort felt during RE. Furthermore, this investigation will provide insight into whether CHO-PRO or $\mathrm{CHO}$ availability will influence the perception of effort during resistance training. In addition to an attenuated RPE response, a similar reduced postexercise salivary cortisol response due to supplementation may improve potential muscular adaptations and enhance recovery following RE sessions. Collectively such potential benefits of $\mathrm{CHO}-\mathrm{PRO}$ or $\mathrm{CHO}$ supplementation can improve performance during training, enhance recovery, and contribute to improved sport performance. 


\subsection{LITERATURE REVIEW}

\subsection{ACUTE RESPONSES TO RESISTANCE EXERCISE}

\subsubsection{Physiological}

Resistance exercise can stimulate a number of acute physiological responses in the body. These responses can include: 1) decreases in blood glucose and muscle glycogen concentrations; 2) increases in anabolic and catabolic hormones; 3) changes in the balance of muscle protein synthesis and degradation; and 4) onset of central and peripheral fatigue. Collectively, these physiological responses are important because they directly affect exercise performance, recovery from exercise, and muscular adaptations to a resistance exercise session. The following sections will explore these mechanisms.

\subsubsection{Blood Glucose/Muscle Glycogen}

The phosphagen system (ATP-PC) has been considered a primary source of energy during short-duration, high intensity exercise, with glycogenolysis and glycolysis contributing minimally (Haff, Lehmkuhl, McCoy, \& Stone, 2003). However, numerous 
research studies have shown that muscle glycogen stores can become significantly decreased during resistance exercise (Haff et al., 2000; MacDougall et al., 1999; Pascoe et al., 1993; Robergs et al., 1991; Tesch et al., 1996, 1998). An investigation by Tesch et al. (1998) found a $40 \%$ reduction in muscle glycogen after 5 sets of 10 repetitions of concentric knee extensions performed at $60 \%$ of $1 \mathrm{RM}$, and a $20 \%$ reduction after 5 sets of 10 repetitions at $45 \%$ of 1 RM. In a similar study, Robergs et al. (1991) found a 39\% reduction in muscle glycogen after 6 sets of 6 repetitions of leg extensions performed at 70\% of 1 RM. Pascoe et al. (1993) demonstrated a 31\% reduction in muscle glycogen after leg extensions performed to muscular failure. Finally, a study by Haff et al. (2000) showed a $26.7 \%$ decrease in muscle glycogen as a result of a multiple set resistance exercise session consisting of 3 different leg squats.

In general, these studies have demonstrated that an acute resistance exercise session can result in a $20-40 \%$ decrease in muscle glycogen stores depending on the intensity, duration, and volume of work used during the exercise session. Additionally, Robergs et al. (1991) demonstrated that the volume of work performed during an acute resistance exercise session significantly affects the amount of glycogenolysis, while the intensity significantly affects the rate of glycogenolysis. Therefore, moderate to high intensity, high volume resistance exercise that is commonly performed by many athletes has the potential to stimulate significant glycogenolysis. Decreased muscle glycogen can have detrimental effects on resistance exercise performance including increased muscular weakness, decreased maximal muscular strength, and decreased maximal force production (Yaskpelkis et al., 1993; Jacobs et al., 1981; Hepburn et al., 1982). 


\subsubsection{Hormonal Responses}

Resistance exercise has been shown to elicit acute hormonal responses in athletes (Beaven, 2008) and recreationally weight trained males when performed at a moderate intensity and high volume (Crewther, 2008). Acute increases in anabolic hormones such as insulin, growth hormone, and testosterone function to enhance protein synthesis in skeletal muscle and promote muscle growth. Acute increases in catabolic hormones such as cortisol stimulate muscle protein breakdown in an attempt to provide energy in response to declines in muscle glycogen and blood glucose (Beaven, 2008).

\subsection{Cortisol}

Cortisol is an adreno-cortical steroid hormone released into the body from the adrenal cortex in response to stressful physical or psychological stimuli (Fleck and Kraemer, 2004). Cortisol levels follow a daily pattern that is affected by circadian rhythms, eating and exercise. In general, cortisol levels are higher during the morning and decline later in the day, yet can increase in response to exercise and food intake (Brooks et al., 2004). Cortisol helps to provide energy in response to decreases in muscle glycogen and blood glucose by stimulating gluconeogenesis and peripheral release of substrates. In peripheral tissue, cortisol stimulates lipolysis in adipose cells and increases protein degradation and decreases protein synthesis in muscle cells. These actions result in elevated triglycerides and amino acids in circulation to be used for energy production (Fleck and Kraemer, 2004). Additionally, some studies suggest cortisol may be a 
mediator of perceived exertion during exercise because it exhibits a positive correlation with RPE (Utter et al., 2007).

\subsection{Salivary Cortisol Measures}

Salivary cortisol sampling is a non-invasive method to measure cortisol, considered advantageous, because it can eliminate hormonal shifts due to the stress response (i.e. anxiety) associated with blood sampling techniques (Crewther, 2008). For a valid and reliable salivary cortisol measure to occur, it needs to accurately reflect the concentration of serum cortisol. Numerous studies have examined the validity and reliability of salivary cortisol compared to serum cortisol and found correlations ranging from $r=0.85$ to 0.97 (Gallagher et al., 2006; Peters et al., 1982; Umeda et al., 1981).

Gozansky et al. (2005) showed that salivary cortisol was significantly correlated $(r=0.89)$ with serum free cortisol. Furthermore, it was suggested that salivary cortisol may actually provide a better measure than serum cortisol because of its accuracy in measuring unbound cortisol. Unbound cortisol is not bound to cortisol-binding globulin (CBG) or other proteins and may provide more relevant data in regards to physical activity.

\subsection{Salivary Cortisol and Resistance Exercise}

A number of studies have examined the acute salivary cortisol response to resistance exercise. Crewther et al. (2008) found that an acute hypertrophy resistance exercise 
protocol consisting of 10 sets of 10 repetitions of the squat exercise at $75 \%$ of 1 repetition maximum resulted in a significant post-exercise increase in salivary cortisol (Figure 1). Compared to pre-exercise concentrations, cortisol increased by $47 \%$ immediately postexercise and increased up to $290 \%$ over pre-exercise concentrations at 45 minutes postexercise. In contrast, no increases in salivary cortisol were discovered post-exercise for either a power resistance exercise protocol consisting of 8 sets of 6 repetitions at $45 \%$ of 1 repetition maximum or a maximal strength scheme consisting of 6 sets of 4 repetitions at $88 \%$ of 1 repetition maximum.

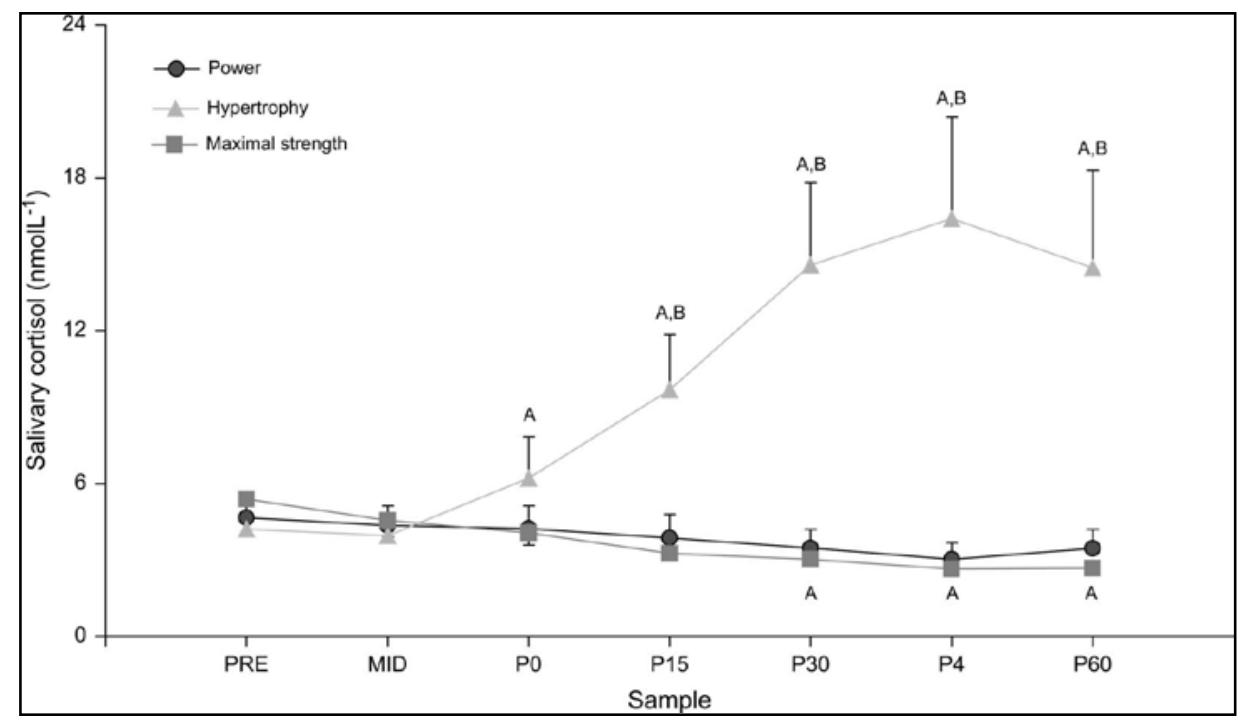

Figure 1: Salivary cortisol response to various intensity resistance exercise protocols (Crewther et al., 2008).

An investigation by McGuigan et al. (2004) examined the effects of high and low intensity resistance exercise at 75 and $30 \%$ of 1 repetition maximum, respectively, on salivary cortisol responses. The high intensity resistance exercise protocol consisted of 6 
sets of 10 repetition squats and 6 sets of 10 repetition bench press performed at $75 \%$ of 1 repetition maximum, while the low intensity protocol consisted of 3 sets of 10 repetitions at $30 \%$ of 1 repetition maximum of the same exercises. Both protocols utilized 2 minute rest periods between sets. They found a 97\% increase in salivary cortisol immediately following the high intensity resistance exercise protocol, while there was no significant change following the low intensity protocol. There was also a significant immediate post-exercise difference (145\%) in salivary cortisol responses between the high and low protocols.

It appears there is strong evidence to suggest moderate to high intensity, high volume resistance exercise can cause significant increases is salivary cortisol. This type of workout protocol is typically utilized by recreational weight lifters who are concerned about muscular hypertrophy. On the other hand, it appears that low intensity, high volume resistance exercise and high intensity, low volume resistance exercise does not show any significant increases in salivary cortisol.

\subsubsection{Muscle Protein Synthesis/Degradation}

Resistance exercise stimulates the process of building muscle protein from amino acids (protein synthesis), and breaking down muscle protein into amino acids (protein degradation) (McArdle, Katch, Katch, 2001). The balance of protein synthesis and protein degradation determines the muscle tissue building response (i.e., anabolic response) to resistance exercise (Volek, 2004). Hypertrophy of skeletal muscle can occur as a result of an increase in protein synthesis, a decrease in protein degradation, or a 
combination of both. Following an acute bout of resistance exercise, muscular hypertrophy can occur if there is a positive net protein balance. A positive net protein balance occurs when the amount of protein synthesized exceeds the amount of protein degraded (Fleck and Kraemer, 2004).

Phillips et al. (1997) found that a leg extension resistance exercise protocol consisting of 8 sets of 8 repetitions at $80 \%$ of 1 RM increased protein synthesis by $112 \%$ at 3 hours post-exercise, $65 \%$ at 24 hours post-exercise, and $34 \%$ at 48 hours postexercise. They also found that protein degradation was elevated by $31 \%, 18 \%$, and $1 \%$ at 3, 24, and 48 hours post-exercise. These results suggest the net protein balance was elevated by 23 to $48 \%$ over the 48 hour post-exercise time period with a majority occurring during the first 3 hours post-exercise. A similar study by MacDougall et al. (1995) demonstrated an increase in muscle protein synthesis that lasted up to 24 hours following resistance exercise, but returned to baseline by 36 hours post-exercise.

There are a number of factors that influence net protein balance including training status, hormonal regulation, amino acid availability, and timing of nutritional intake. An investigation by Phillips et al. (1999) examined protein synthesis and degradation in resistance trained and untrained men. They discovered that the rate of protein synthesis was higher in the untrained (118\%) compared to the trained men (48\%) at 4 hours postexercise. However, the rate of degradation was also higher in the untrained men resulting in no significant differences in positive net protein balance between trained (37\%) and untrained men (34\%).

In the fasted state, muscle protein degradation exceeds muscle protein synthesis, resulting in a net negative muscle protein balance. Resistance exercise alone has been 
shown to increase muscle protein synthesis and degradation, such that net muscle protein balance remains negative (Biolo et al. 1995). An investigation by Biolo et al. (1997) showed resistance exercise followed immediately by amino acid intake resulted in a positive net muscle protein balance. The positive net muscle protein balance was due to increased amino acid availability and muscle blood flow after resistance exercise which caused an approximate doubling of the rate of protein synthesis. Additionally, studies have shown that carbohydrate intake after resistance exercise results in elevated insulin concentrations. Elevated insulin concentrations after resistance exercise caused no significant change in muscle protein synthesis, but did reduce exercise-mediated muscle protein degradation resulting in a positive net muscle protein balance (Biolo et al., 1999; Roy et al., 1997).

Furthermore, a study by Tipton et al. (2001) demonstrated that carbohydrate and amino acid intake before resistance exercise results in an even greater increase in the rate of protein synthesis compared to intake post-exercise. The investigators suggest that the combination of amino acids (to increase amino acid availability) and carbohydrates (to stimulate insulin release) can be a potent stimulator of net muscle protein synthesis. When the supplement was ingested post-exercise, net muscle protein balance and phenylalanine $R_{d}$, an index of muscle protein synthesis, was unchanged. On the other hand, when the supplement was ingested before exercise, phenylalanine $R_{d}$ and net muscle protein balance were increased. The increased protein synthesis is due to optimal amino acid delivery and transport to the muscle by the increased blood flow during the resistance exercise session. 
Tipton and Wolfe (1998) developed a model of protein metabolism during resistance exercise that proposed: 1) resistance exercise stimulates protein synthesis, 2) intra-cellular amino acid concentrations are reduced, 3) decreased amino acid concentrations stimulate protein breakdown or the transport of amino acids into the muscle cell, 4) increased availability of amino acids from protein breakdown or transport into the cell further stimulates protein synthesis resulting in tissue remodeling. Based on this model of protein metabolism, Tipton and Wolfe (1998) suggest that availability of amino acids inside the muscle cell during and following resistance exercise may be a limiting factor for protein synthesis. The exercise-induced stimulation of protein synthesis causes a decrease in intracellular amino acids and without an increase in protein breakdown or amino acid transport into the cell protein synthesis will be limited. Therefore, the timing of protein intake following exercise may be critical to post-exercise recovery.

\subsubsection{Central and Peripheral Fatigue}

During exercise physical fatigue can occur within skeletal muscle, known as peripheral fatigue, or within the central nervous system, known as central fatigue. Peripheral fatigue occurs due to a number of biochemical mechanisms including depletion of phosphocreatine, blood glucose, and glycogen within the muscle (Blomstrand, 2001). Phosphocreatine is the immediate source for the rephosphorylation of adenosine triphosphate (ATP), which is the high energy intermediate that supports muscle contraction (Brooks et al., 2005). ATP and phosphocreatine are stored in small amount 
with the muscle and supply energy for muscular contractions during maximal or near maximal intensity physical activity. If high intensity physical activity continues for longer than 15 to 20 seconds, fatigue will occur when phosphocreatine stores within the muscle are depleted and ATP production is significantly decreased (Housh et al., 2006)

The aerobic metabolism of fat and glycogen stored within the liver and muscle are used to produce energy for moderate intensity, long-duration physical activity. Over the course of long-duration physical activity glycogen stores are gradually depleted within the working muscles and can lead to fatigue even though there is unlimited potential energy from stored fat (Housh et al., 2006). Blood glucose is released by the liver in response to energy needs during physical activity but is limited by glycogen stores within the liver and the process of gluconeogenesis. Therefore, blood glucose production may fall below that required by working muscles and in combination with depleted muscle glycogen result in fatigue (Brooks et al., 2005).

In addition, peripheral fatigue can also occur due to the accumulation of metabolites such as lactate, inorganic phosphate, and ammonia within the muscle and blood. Along with the accumulation of lactate, there is also an increase in hydrogen ions and acidity and decrease in $\mathrm{pH}$ in the muscle. Increased lactate accumulation means that the mechanisms of lactate removal have been exceeded and the body is failing to cope with the metabolic demands of the exercise session. The build-up of lactate interferes with muscle contraction by affecting the following: 1) calcium release from the sarcoplasmic reticulum, 2) actin-myosin binding, 3) ATP breakdown, 4) ATP production (Housh et al., 2006). 
Research has shown that the primary cause of fatigue during high-intensity exercise may not be lactate, but rather the build-up of inorganic phosphate in the muscle due to the breakdown of creatine phosphate for the production of ATP. Inorganic phosphate accumulation may lead to fatigue by affecting a number of cellular processes including: 1) actin-myosin binding, 2) myofibrillar calcium sensitivity for binding with troponin, 3) calcium release from the sarcoplasmic reticulum, 4) calcium uptake by the sarcoplasmic reticulum (Westerblad et al., 2002).

While the mechanisms of peripheral fatigue have been well indentified, the mechanisms of central fatigue are not known. Central fatigue may be due to the inhibition of neural drive as a result of impaired sensory feedback from working muscles or the effects of either exercise-induced accumulation of the metabolite ammonia or an increase in the neurotransmitter serotonin in the brain (Housh et al., 2006). Exercise increases ammonia production which can alter the levels of neurotransmitters and ATP within the brain and contribute to the perception of fatigue by causing deep and labored breathing. Serotonin accumulation in the brain during prolonged exercise can impair the central nervous system. This build-up of serotonin results from increased transport of the amino acid tryptophan across the blood-brain barrier during exercise (Housh et al., 2006).

Blomstrand (2001) suggests that plasma amino acid concentrations may affect central fatigue by influencing the synthesis, concentration, and release of neurotransmitters in the brain (Table 1). The main neurotransmitter that is thought to be affected is 5-hydroxytryptamine (5-HT) which is involved in the control of arousal, sleepiness and mood. Plasma tryptophan is the precursor for the synthesis of 5-HT in the brain. The rate-limiting step in the synthesis of 5-HT is the transport of tryptophan across 
the blood-brain barrier into the brain. Tryptophan and branched-chain amino acids can compete to cross the blood-brain barrier. Therefore, the amount of tryptophan transported into the brain depends on the concentration of tryptophan in the blood and the concentration of branched-chain amino acids in the blood. Research by Blomstrand et al. (1997) showed that the plasma ratio of free tryptophan/BCAA increases during and after exercise which means that more tryptophan can be transported into the brain. This elevated tryptophan in the brain causes the synthesis and release of 5-HT which may lead to central fatigue.

\section{Table 1: A theory to explain the cause of central fatigue during exercise} (Blomstrand, 2001).

\begin{tabular}{|l|l|l|}
\hline Uptake of BCAA by the muscle & increases & \\
Plasma level of BCAA & decreases & \\
Release of free fatty acids & increases & Plasma ratio free tryptophan \\
Plasma free tryptophan & increases & and BCAA increases \\
Transport of tryptophan & increases & \\
Synthesis and release of 5-HT & increase & Causes fatigue \\
\hline
\end{tabular}

\subsubsection{Perceptual}

\subsubsection{Ratings of Perceived Exertion (RPE)}

Perceived exertion is defined as the subjective feeling of effort, strain, discomfort, and fatigue that a person experiences during exercise (Robertson and Noble, 1997). At the onset of exercise, physiological, psychological, and symptomatic mediators are integrated 
to create the sensation of effort, strain, discomfort, or fatigue throughout an effort continuum. Borg's model of the effort continua suggests the subjective response to an exercise stimulus involves three main effort continua: physiological, perceptual and performance (Borg, 1982). The effort continua demonstrate a relationship between the physiological demands and perception of an exercise performance. The functional link between the three effort continua suggest that perceptual responses provide similar information about exercise performance as do selected physiological responses. Therefore, decisions about intensity and duration of exercise can be based on the functional interaction between the perceptual and physiological continua (Robertson and Noble, 1997).

\subsubsection{Physiological Mediators}

The physiological mediators that influence perceived exertion are classified as peripheral, respiratory-metabolic, and nonspecific (Robertson, 2004). Peripheral physiological mediators are localized in the limbs and trunk and consist of blood $\mathrm{pH}$, lactic acid, blood glucose, muscle blood flow, muscle fiber type, free fatty acids, and muscle glycogen. Respiratory-metabolic mediators are physiological responses that influence ventilatory drive during exercise and include: pulmonary ventilation $\left(\mathrm{V}_{\mathrm{E}}\right)$, oxygen uptake $\left(\mathrm{VO}_{2}\right)$, carbon dioxide production, heart rate (HR), and blood pressure (BP). The nonspecific mediators are generalized or systemic physiological events that occur during exercise, and consist of changes in catecholamine levels, body temperature, pain, cortisol, serotonin, and cerebral blood flow (Robertson, 2004). 
Carbohydrate availability (blood glucose and muscle glycogen) is considered a mediator of perceived exertion during prolonged aerobic endurance exercise (Robertson et al., 1990; Utter et al., 1999, Utter et al., 2007). During prolonged aerobic endurance exercise, there is a decrease in blood glucose concentration which increases the reliance on muscle glycogen to produce ATP. As muscle glycogen levels decrease, there is an interruption in the supply of energy for muscular contraction which results in fatigue. In order for fatiguing muscle to maintain a constant power output, motor unit recruitment and firing frequency must increase which in turn will increase perceived exertion (Robertson et al., 1990).

A number of studies have suggested a potential link between perceived exertion and cortisol levels during exercise. Utter et al. (1999) established a potential association between insulin, cortisol, and RPE in an investigation to determine the effect of carbohydrate supplementation during 2.5 hours of high-intensity running and cycling performed at 75\% maximum oxygen uptake. Higher levels of insulin and lower levels of cortisol were associated with lower ratings of perceived exertion and higher levels of plasma glucose due to supplementation. Backhouse et al. (2005) investigated the effect of carbohydrate supplementation on perceived exertion during prolonged cycle exercise. Plasma cortisol levels and ratings of perceived exertion were significantly higher for the placebo group compared to the carbohydrate group. Additionally, cortisol and perceived exertion were positively correlated.

Hollander et al. (2003) compared perceived exertion and cortisol responses with concentric and eccentric resistance exercise protocols using the same absolute workload. The concentric resistance exercise protocol resulted in significantly greater ratings of 
perceived exertion (6.71 \pm 0.51 and $4.10 \pm 0.27$, respectively) and cortisol responses than the eccentric resistance exercise protocol. The investigators also determined that there was a significant correlation $(\mathrm{r}=0.51, \mathrm{p}<0.05)$ between the last rating of perceived exertion during both resistance training protocols and cortisol measured immediately post-exercise.

\subsubsection{RPE during Resistance Exercise}

Ratings of perceived exertion are commonly used to regulate and prescribe resistance exercise intensity. Investigators have demonstrated that RPE is a valid method for determining differences in resistance exercise intensity (Suminski et al., 1997; Gearhart et al., 2002; Lagally et al., 2002, 2004). These investigators established concurrent validity by showing significance between \% of 1-RM lifted and RPE as measured by the Borg CR-10 scale. Additionally, concurrent validity has also been established using other criterion variables such as blood lactic acid concentrations and muscle activity as measured by electromyography (Lagally et al., 2002, 2004)

Suminski et al. (1997) found that the RPE for the overall body, measured on the Borg CR-10 scale, was significantly higher during exercise at 70\% 1-RM than at 50\% 1RM (8.8 \pm 0.7 vs. $5.5 \pm 0.6)$. This demonstrated that the Borg CR-10 scale could be used to monitor resistance exercise intensity and provide information on the relative $\%$ of 1 RM lifted. Gearhart et al. (2002) showed that RPE for active muscle (RPE-AM) was significantly higher for an acute bout of resistance exercise at 90\% of 1-RM than at 30\% of 1-RM when volume of work was held constant $(14.11 \pm 2.08$ vs. $8.34 \pm 1.35$ for bench 
press exercise). This demonstrated that higher ratings of perceived exertion are attributed to greater force production by the active muscle and not greater volume of work. Based on the findings of these investigations, RPE can be used to accurately determine differences in resistance exercise intensity.

The OMNI Perceived Exertion Scale for Resistance Exercise (OMNI-RES) was developed by Robertson et al. (2003) and has been validated by a number of studies. The OMNI-RES has both verbal and non-specific pictorial descriptors to make the scale easier for health-fitness practitioners to use (Figure 3). Concurrent validity of the OMNIRES was established using volume of work and blood lactic acid concentration as criterion variables. Subjects performed bicep curl and knee extension exercises at $65 \%$ of 1-RM intensity and rated perceived exertion for the active muscle and overall body. Results showed a positive association between RPE and volume of work and blood lactic acid concentration during upper and lower body resistance exercise $(r=0.79$ to $0.91, \mathrm{p}<$ 0.01). This demonstrates concurrent validity of the OMNI-RES for use by young recreationally active males and females during resistance exercise (Robertson et al., 2003).

Construct validity was established by correlating RPE from the OMNI-RES with RPE from the Borg Scale over a range of resistance exercise intensities. Subjects performed 1 repetition of the knee extension exercise at 40, 50, 60, 70, 80, and 90\% of 1 RM and perceived exertion for the active muscle and overall body was measured using both the Borg and OMNI-RES scales. Results showed a positive linear relationship between RPE from the Borg scale and OMNI-RES scale ( $\mathrm{r}=0.94$ to $0.97, \mathrm{p}<0.01$ ) (Lagally and Robertson, 2006). Based on the results from these studies, the OMNI-RES 
can be considered a valid tool for measuring perceived exertion during resistance exercise.

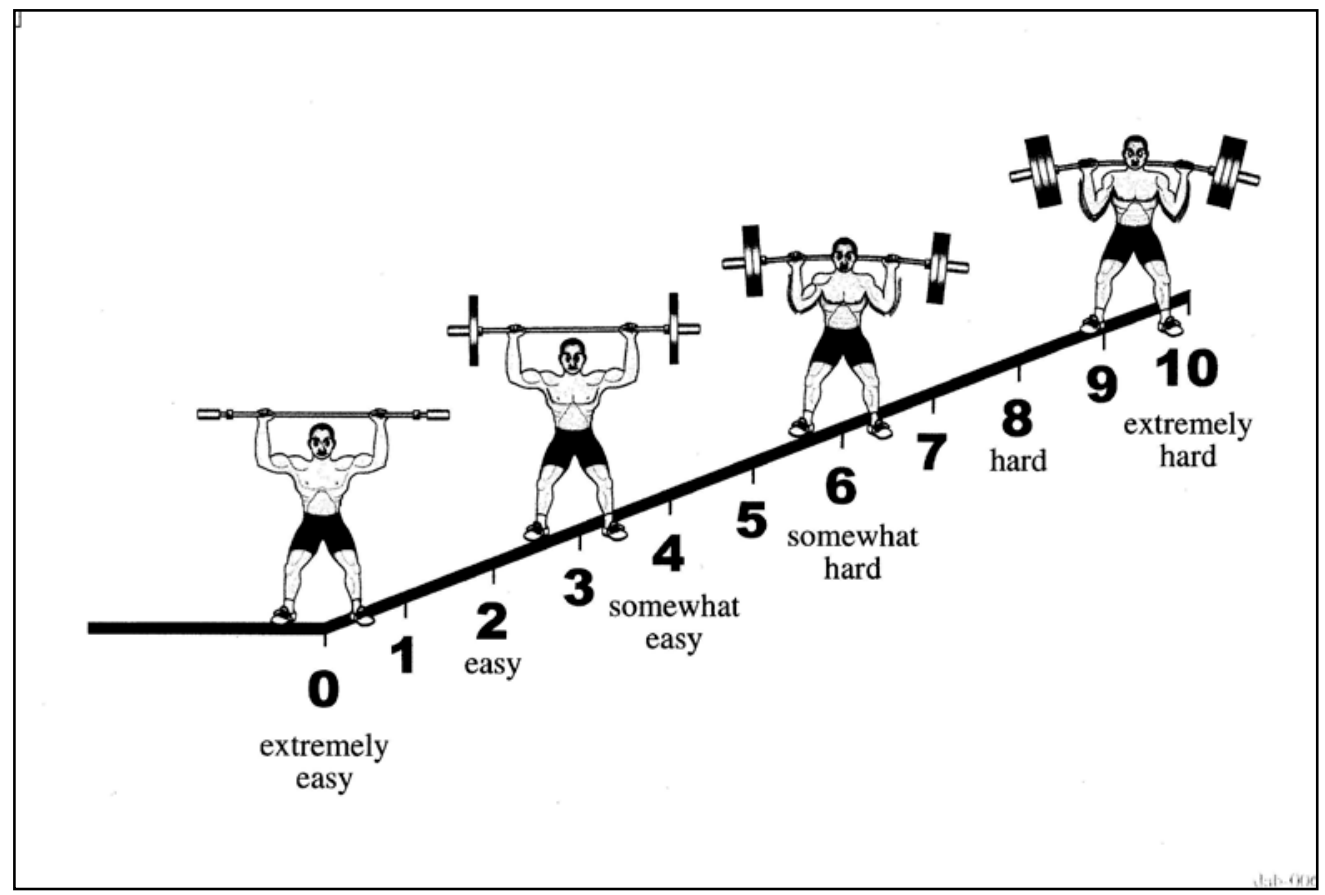

Figure 2: OMNI perceived exertion scale for resistance exercise (OMNI-RES).

\subsubsection{Session RPE}

Session RPE is defined as the post-exercise rating of global exertion experienced during an entire exercise session and was introduced and validated by Foster et al. (2001). The session RPE allows for a single rating of perceived exertion for an entire exercise session, rather than reporting a series of RPE measures during an exercise session. The goal for session RPE is to simplify the monitoring of aerobic and resistance exercise intensity for fitness professionals (McGuigan et al., 2008). 
Foster et al. (2001) compared session RPE with the summated heart rate zone method of quantifying aerobic exercise and found the two methods were highly correlated. Sweet et al. (2004) applied the session RPE method to resistance training and found it a viable method for quantifying the intensity of resistance training. Session RPE increased from $3.86 \pm 1.6$ to $5.76 \pm 1.7$ to $6.36 \pm 1.4$ as the percentage of $1 \mathrm{RM}$ increased from $50 \%$ to $70 \%$ to $90 \%$, respectively. A study by Egan et al. (2006) found no significant difference between the average RPE measured during resistance exercise and the session RPE obtained 30 minutes post-exercise.

A similar study by Day et al. (2004) evaluated the effectiveness and reliability of the session RPE method as a tool to quantify exercise intensity during resistance exercise sessions. Subjects performed high, moderate, and low intensity resistance exercise protocols. RPE was measured following the completion of each set and 30 min postexercise (session RPE). The investigators found that the session RPE for the high intensity protocol was significantly greater than the moderate and low intensity protocols. Additionally, the session RPE was not significantly different from the average RPE measured during the exercise session. Results of these investigations suggest that session RPE is a valid and reliable method to quantify resistance exercise intensity. Therefore, it is of interest to determine if $\mathrm{CHO}-\mathrm{PRO}$ and $\mathrm{CHO}$ supplementation during resistance exercise will have any attenuating effects on session RPE following a resistance exercise session. 


\subsection{CARBOHYDRATE SUPPLEMENTATION}

Carbohydrate supplementation during exercise in the form of CHO-electrolyte beverages has become a popular practice among recreational and professional athletes. $\mathrm{CHO}$ supplementation during an exercise session can prevent hypoglycemia and provide a fuel source which is immediately usable by working muscles to help improve physical work capacity (i.e. ergogenic effect). Research has shown that CHO supplementation can increase time to exhaustion and improve performance times during prolonged aerobic endurance events. Additionally, ingesting $\mathrm{CHO}$ and electrolytes in a liquid form helps to offset body fluid losses from sweating during exercise to help prevent dehydration (Tsintzas and Williams, 1998).

\subsubsection{Possible Mechanisms for Acute Responses}

Tsintzas and Williams (1998) suggest two physiological/metabolic mechanisms to explain the ergogenic effect of $\mathrm{CHO}$ ingestion during exercise. The first mechanism is the restoration of euglycemia and increased oxidation of blood glucose late in exercise when muscle glycogen utilization is diminished. The second mechanism is a decreased rate of muscle glycogen utilization, which delays the depletion of muscle glycogen and the point at which fatigue is reached. The reliance on either mechanism depends on factors such as type and intensity of exercise, and the type and timing of CHO ingestion.

The amount and timing of $\mathrm{CHO}$ ingestion may play an important role in determining the effect of $\mathrm{CHO}$ ingestion on muscle glycogen utilization. The ingestion 
of 5 to $8 \mathrm{ml} \mathrm{CHO}$ solution per kg body mass immediately before exercise, followed by ingestion of $2 \mathrm{ml}$ per $\mathrm{kg}$ body mass every 15 to 20 minutes has been shown to stimulate a fast rate of gastric emptying. This supplementation protocol results in increased blood glucose, increased plasma insulin, and decreased muscle glycogen response during the first hour of exercise. On the other hand, when $\mathrm{CHO}$ is ingested 10 to 30 minutes after the initiation of exercise a glycogen-sparing effect is not observed.

During continuous cycling of moderate intensity, $\mathrm{CHO}$ ingestion results in minimal changes to plasma insulin levels and it appears that enhanced exercise performance is attributed to the ability to maintain blood glucose oxidation. Conversely, during constant pace running, $\mathrm{CHO}$ ingestion results in a sparing of glycogen in type I muscle fibers early in exercise. During intermittent exercise which allows for periods of low intensity exercise or rest periods, $\mathrm{CHO}$ ingestion also results in a sparing of muscle

glycogen. At the same relative exercise intensity $\left(70 \% \mathrm{VO}_{2 \max }\right)$, $\mathrm{CHO}$ ingestion during cycling results in less changes in blood glucose and plasma insulin levels compared with running. Therefore, the intensity and type of exercise, as well as the type and timing of $\mathrm{CHO}$ ingestion are factors in determining the responses of blood glucose, plasma insulin, and muscle glycogen when $\mathrm{CHO}$ is ingested during exercise (Tsintzas and Williams., 1998).

\subsubsection{Effect on Aerobic Exercise}

Numerous studies have demonstrated that carbohydrate supplementation can increase the duration and amount of work performed during an aerobic exercise session (Tsintzas et 
al., 1996; Yaspelkis et al., 1993; Wright et al., 1991; Neufer et al., 1987). Tsintzas et al. (1996) examined the effect of a 5.5\% CHO solution on recreational runners during a run to exhaustion at $70 \% \mathrm{VO}_{2 \max }$. The subjects ingested approximately $48 \mathrm{~g}$ of carbohydrate within the first hour of the run and water was ingested for the remainder of the run to exhaustion. The participants improved their time to exhaustion by $14 \%$ compared with the control trial, in which water was ingested for the entire run. The investigators suggest that $\mathrm{CHO}$ supplementation during the first hour of exercise at $70 \% \mathrm{VO}_{2 \max }$ delays the onset of fatigue by sparing glycogen utilization in the type I fibers of the working muscles.

Wright et al. (1991) examined the affect of no carbohydrate, pre-exercise carbohydrate, carbohydrate during exercise, and the combination of carbohydrate before and during cycling at $70 \%$ of maximal oxygen uptake until exhaustion. Pre-exercise supplementation occurred 3 hours prior to exercise, and consisted of either a $25 \%$ carbohydrate solution supplying 5 g CHO per kg body mass, or a similarly flavored, colored, and textured placebo. Supplementation during exercise occurred every 20 minutes and consisted of either an $8 \%$ carbohydrate solution supplying $0.2 \mathrm{~g} \mathrm{CHO} / \mathrm{kg}$ body mass or a similarly flavored, colored, and textured placebo. Results indicated that total work produced during exercise was 19 to $46 \%$ higher when carbohydrates were consumed, and time to exhaustion was 18 to $44 \%$ greater than when a placebo was consumed.

Yaspelkis et al. (1993) investigated the affect of a 10\% liquid CHO supplement ingested every 20 minutes during low intensity (45\% of $\mathrm{VO}_{2 \max }$ ) and moderate intensity (75\% of $\mathrm{VO}_{2 \max }$ ) continuous cycling exercise. The investigators found that muscle 
glycogen, plasma insulin, and plasma glucose levels were significantly greater during $\mathrm{CHO}$ supplementation compared to a placebo. Additionally, time to fatigue at $80 \%$ of maximal oxygen uptake was significantly greater during $\mathrm{CHO}$ supplementation than during the placebo. Results of these studies suggest that aerobic exercise is improved with carbohydrate supplementation due to an increase of blood glucose, and results in a sparing of muscle glycogen for reliance later during the exercise bout.

\subsubsection{Effect on Resistance Exercise}

As previously stated, the phosphagen system (ATP-PC) has traditionally been considered the primary provider of energy during resistance training, with minimal energy supplied by muscle glycogen. Because resistance exercise typically lasts less than 30 seconds, the ATP-PC system provides energy for the first 5 to 15 seconds of exercise before other energy systems need to assist. Recently, researchers have demonstrated that muscle glycogen stores can significantly decrease by approximately 20 to $40 \%$ during moderate intensity, high volume resistance exercise ranging from 36 to 50 total repetitions performed at 35 to 70\% of maximal strength (Haff et al., 2000; MacDougall et al., 1999; Pascoe et al., 1993; Robergs et al., 1991; Tesch et al., 1996, 1998).

Since muscle glycogen is an important fuel source during moderate intensity, high volume resistance exercise, muscle glycogen decrements may result in fatigue and decreased performance (Yaspelkis et al., 1993; Jacobs et al., 1981; Hepburn et al., 1982). Haff et al. (2003) suggests that the use of a carbohydrate supplement may prevent muscular fatigue and decreases in the volume of work performed during a resistance 
exercise session. As a result, increased muscle glycogen resynthesis may allow athletes to train at higher intensities and perform more work, the result of which may be an enhanced physiological adaptation to resistance exercise.

Several studies have shown that carbohydrate supplementation can attenuate muscle glycogen losses and improve performance during acute resistance exercise (Haff et al., 2001; Haff et al., 1999; Lambert et al., 1991). Lambert et al. (1991) reported that a $10 \%$ CHO supplement ingested immediately prior to exercise and after every fifth set during resistance exercise enhanced performance of 10 repetition sets of leg extensions performed at $80 \%$ of $10-\mathrm{RM}$ to muscular failure. The $\mathrm{CHO}$ supplement resulted in an increased volume of work by increasing the number of sets (+2.7) and repetitions (+20) performed during exercise. A similar study by Haff et al. (2001) showed that a $20 \%$ CHO supplement $(0.3 \mathrm{~g} / \mathrm{kg}$ body mass) ingested immediately before exercise and after every other set during exercise increased the amount of work performed during 16 sets of 10 repetitions of isokinetic leg extensions.

Research has also demonstrated that $\mathrm{CHO}$ supplementation can significantly increase resistance exercise performance when multiple training sessions occur on the same day. In a study by Haff et al. (1999), subjects performed sets of 10 back squats repetitions at 55\% of 1-RM to volitional fatigue during both a morning and afternoon session separated by a 4-hour recovery period. The subjects ingested either a $20 \% \mathrm{CHO}$ solution ( $0.3 \mathrm{~g} / \mathrm{kg}$ body mass) or placebo immediately prior to exercise, every 15 minutes during exercise, and once an hour during a 4 hour recovery period between sessions. The CHO group performed significantly more repetitions (+67.7) and sets (+7.4) and 
increased exercise duration (+31.6 min) during the afternoon session compared to the placebo group.

Conflicting results were reported in a study by Kulik et al. (2008) who examined the effect of a $20 \% \mathrm{CHO}$ supplement ( $0.3 \mathrm{~g} / \mathrm{kg}$ body mass) ingested immediately before exercise, and after every other set during squat exercise performed in sets of 5 repetitions at $85 \%$ of 1-RM to volitional fatigue. They found no significant difference in total sets, repetitions, volume load, and total work performed between the $\mathrm{CHO}$ and placebo groups. The investigators noted that other studies reporting improvements in resistance training performance with $\mathrm{CHO}$ supplementation have utilized protocols with intensities less than 85\% 1RM, durations of exercise longer than 55 minutes, and large workloads.

It appears that an ergogenic effect of carbohydrate supplementation can occur during resistance exercise sessions consisting of a high volume and moderate intensity 70\% 1 RM) which generally last longer than 55 minutes. Haff et al., (2000) suggests that the duration and total volume of work of a resistance exercise session is significant in determining the amount of glycogenolysis that may occur in response to an exercise session. During resistance exercise there seems to be an increased reduction in muscle glycogen and greater utilization of blood glucose directly related to duration (> $55 \mathrm{~min}$ ) and total volume of work performed. Therefore, it appears that the duration and total volume of work plays a significant role in determining the ergogenic "effectiveness" of carbohydrate supplementation during resistance exercise (Kulick et al., 2008).

It is possible that $\mathrm{CHO}$ supplementation may influence the endocrine response to resistance exercise. CHO supplementation enhances anabolic characteristics that could potentially increase muscle hypertrophy and influence resistance exercise performance 
(Haff et al., 2003). An investigation by Roy et al. (1997) demonstrated that following exercise, elevated insulin concentrations due to carbohydrate supplementation resulted in a reduction of exercise-induced protein degradation and a 36\% increase in muscle protein synthesis. Kraemer et al. (1998) reported suppressed cortisol levels and increased growth hormone concentration measured after three days of $\mathrm{CHO}$ supplementation during a heavy resistance training protocol. These results suggest an insulin mediated suppression of cortisol and increased growth hormone concentration due to $\mathrm{CHO}$ supplementation during resistance exercise results in an enhanced anabolic environment. Therefore, it is possible that an enhanced anabolic environment due to $\mathrm{CHO}$ supplementation can potentially increase muscle hypertrophy to a greater extent than resistance exercise without supplementation.

\subsubsection{Effect on Perceived Exertion}

Research has shown that availability of carbohydrate energy is a limiting factor during long duration aerobic exercise (Yaspelkis et al., 1993; Wright et al., 1991; Neufer et al., 1987). Recent investigations have identified a physiological link between ratings of perceived exertion and carbohydrate availability during prolonged aerobic exercise (Noble and Robertson, 1996; Utter et al., 2007). As muscle glycogen levels decrease, there is an interruption in the supply of energy for muscular contraction which results in fatigue. In order for fatiguing muscle to maintain a constant power output, motor unit recruitment and firing frequency must increase which in turn can increase perceived exertion (Robertson et al., 1990). 
It is plausible that carbohydrate supplementation before and during prolonged exercise can aid the maintenance of blood glucose levels and spare muscle glycogen which attenuates perceived exertion. However, evidence is limited regarding the ability of carbohydrate supplementation to attenuate ratings of perceived exertion during resistance training. A recent study examining the effect of a $6 \%$ carbohydrate solution ingested 15-30 minutes before exercise $(8 \mathrm{ml} / \mathrm{kg})$ and during exercise $(10 \mathrm{ml} / \mathrm{kg} / \mathrm{h})$ found no attenuating effect on ratings of perceived exertion during a moderate intensity resistance training protocol. This study utilized a 10 exercise total body resistance training protocol in which the subjects performed 4 sets of 10 repetitions for each resistance exercise, with the first set at $40 \%$ of the subject's 1 repetition maximum (RM), and subsequent sets at $60 \%$ of $1 \mathrm{RM}$. This protocol lasted approximately 2 hours, utilized two to three minute rest intervals, and was designed to maximize the total training volume (Utter et al., 2005). The author suggested the use of higher intensity resistance training protocols in future research regarding $\mathrm{CHO}$ supplementation. Other authors have suggested research be done using various intensities, sets, repetitions, and rest intervals due to the large variety of used by recreational and professional athletes.

\subsection{CARBOHYDRATE-PROTEIN SUPPLEMENTATION}

Commercially available sport beverages containing carbohydrate and protein have gained popularity among recreational and professional athletes due to claims suggesting that the

added protein improves performance. Some of these beverages are designed as a 
supplement to be used during exercise, while others have higher nutrient concentrations and are consumed immediately after exercise to facilitate recovery (Romano et al., 2006). To date, limited evidence suggests that these carbohydrate and protein beverages increase performance time to fatigue, reduce post-exercise muscle damage, and enhance muscle glycogen repletion versus carbohydrate-only beverages (Romano et al., 2006; Saunders et al., 2006; Saunders et al., 2004; Ivy et al., 2003).

\subsubsection{Possible Mechanisms for Acute Responses}

There are a number of possible physiological mechanisms responsible for the ergogenic effects of CHO-PRO supplementation during exercise. Research suggests that the addition of protein to a carbohydrate supplement can suppress cortisol and increase the insulin response to a greater extent than carbohydrate alone (Ivy et al., 2003; Baty et al, 2007; Bird et al, 2006a, 2006b, 2006c). This improved hormonal response may help to reduce the significant decrease in muscle glycogen that occurs during exercise, and result in an enhanced anabolic environment where muscular adaptations and recovery can occur. Furthermore, CHO-PRO supplementation increases the availability of amino acids which may result in an increased uptake of amino acids by the muscle. Increased uptake of amino acids by the muscle enhances net muscle protein balance and improves the anabolic environment (Volek, 2004). Additionally, CHO-PRO supplementation provides the greatest attenuation of muscle protein degradation, as evidenced by the reduction of markers such as 3-methylhistidine (Bird et al, 2006a) and creatine kinase (Baty et al, 2007) which may be due to elevated plasma amino acid availability (Baty et al, 2007). 
Another possible mechanism for the ergogenic effect of CHO-PRO supplementation is its potential effect on central fatigue. Evidence has shown that branched-chain amino acids (leucine, isoleucine, and valine) found in most protein supplements may delay central fatigue by influencing the synthesis, concentration and release of neurotransmitters in the brain (Blomstrand et al., 1997). During exercise, plasma branch-chain amino acids decrease and plasma tryptophan increases due to an unloading of tryptophan from albumin. Because tryptophan and branched-chain amino acids compete for the same transporter across the blood-brain barrier, the increase in the ratio of plasma free tryptophan to branched-chain amino acids enhances brain uptake of tryptophan. Tryptophan is a precursor to serotonin, which lowers brain activity and induces central fatigue. CHO-PRO supplementation increases the amount of branchedchain amino acids and reduces the uptake of tryptophan across the blood-brain barrier. The results may reduce central fatigue and improve exercise performance (Blomstrand, 2003).

\subsubsection{Effect on Aerobic Exercise}

Evidence suggests that consumption of a CHO-PRO supplement compared to a $\mathrm{CHO}$ supplement can improve performance, attenuate perceived exertion, and reduce postexercise cortisol levels during aerobic endurance exercise (Saunders et al., 2004; Saunders et al., 2006; Ivy et al., 2003; Romano et al., 2006). Ivy et al. (2003) compared the effects of $\mathrm{CHO}$ and $\mathrm{CHO}-\mathrm{PRO}$ supplementation on aerobic endurance performance in cyclists. Subjects exercised on separate occasion at $45 \%$ of $\mathrm{VO}_{2 \max }$ for 3 hours, $75 \%$ of 
$\mathrm{VO}_{2 \max }$ for 3 hours, and at $85 \%$ of $\mathrm{VO}_{2 \max }$ until fatigue. A $7.75 \% \mathrm{CHO}$ supplement ingested every 20 minutes significantly increased time to exhaustion compared to a placebo $(19.7 \pm 4.6 \mathrm{~min}$ vs. $12.7 \pm 3.1 \mathrm{~min})$, while the addition of $1.94 \%$ protein resulted in an even greater increased time to exhaustion (26.9 $\pm 4.5 \mathrm{~min})$.

Saunders et al. (2004) compared the effect of CHO (7.3\% solution) and CHOPRO (7.3\% CHO, 1.8\% PRO solution) supplements ingested every 15 minutes and immediately post-exercise on endurance cycling performance and post-exercise muscle damage. Subjects performed at $75 \%$ of $\mathrm{VO}_{2 \text { peak }}$ to volitional fatigue, followed by a second ride to exhaustion at $85 \%$ of $\mathrm{VO}_{2 \text { peak }} 12$ to 15 hours later. During the first ride subjects lasted 29\% longer when consuming the CHO-PRO supplement $(106.3 \pm 45.2$ min) compared to the $\mathrm{CHO}$ supplement $(82.3 \pm 32.6)$. During the second ride subjects performed $40 \%$ longer when consuming the CHO-PRO supplement (43.6 \pm 12.5 min) compared to the CHO supplement (31.2 $\pm 8.7 \mathrm{~min})$. Furthermore, post-exercise plasma CPK levels, indicative of muscle damage, were $83 \%$ lower after the CHO-PRO trial compared to the CHO trial.

A similar study by Romano et al. (2006) compared the effects of a CHO-PRO (7.5\% CHO and 1.8\% PRO) supplement to an isocaloric CHO (9.3\% CHO) supplement consumed every 15 minutes and immediately post-exercise on time to fatigue and postexercise muscle damage. In contrast to previous studies, the researchers found no significant difference in time to fatigue between $\mathrm{CHO}-\mathrm{PRO}$ and $\mathrm{CHO}$ supplementation. The CHO-PRO supplement attenuated post-exercise muscle damage compared to the isocaloric CHO supplement, as evidenced by less change in CK and LDH measurements. 
Results of this study indicate there is no difference in endurance performance between CHO-PRO and CHO supplements when they contain the same number of total calories.

\subsubsection{Effect on Resistance Exercise}

Carbohydrate-protein (CHO-PRO) supplementation appears to be more effective than carbohydrate (CHO) supplementation alone or a placebo (PLA) for enhancing muscular adaptations and improving recovery following resistance exercise. Baty et al. (2007) examined whether resistance exercise performance and post-exercise muscle damage were altered when a carbohydrate-protein beverage was consumed. Subjects performed 2 sets of 8 repetitions at their $8 \mathrm{RM}$ and a third set of as many reps possible to volitional fatigue. Results showed that the CHO-PRO supplement did not improve performance during the resistance exercise bout, but appeared to reduce muscle damage, as evidenced by the responses of both myoglobin and creatine kinase. There was a significant increase in cortisol in PLA group at 24 hours post, and insulin concentration significantly increased in CHO-PRO group compared to the CHO group. Results suggest that the use of a CHO-PRO supplement during resistance exercise may reduce muscle damage and enhance recovery.

Bird et al. (2006) examined the influence of 6\% liquid carbohydrate and 6-g essential amino acid supplement ingestion during resistance exercise on the immediate hormonal response and myofibrillar protein degradation as assessed by 3-methylhistidine (3-MH). The resistance exercise protocol consisted of 3 sets of 10 repetitions at 75\% of 1 RM with 1 min of rest between sets and 2 min rest between exercises. The findings 
showed that both $\mathrm{CHO}$ and CHO-EAA ingestion resulted in significantly elevated glucose and insulin concentrations. $\mathrm{CHO}$ and CHO-EAA ingestion resulted in a decrease in cortisol levels of 11 and $7 \%$, respectively. In contrast, the PLA ingestion resulted in a peak cortisol increase of $105 \%$. Both $\mathrm{CHO}$ and EAA ingestion attenuated 3-MH excretion 48 hours after exercise, but the CHO-EAA ingestion resulted in a $27 \%$ reduction in 3-MH excretion, while the PLA ingestions resulted in a 56\% increase in 3MH excretion. These data demonstrate that CHO-EAA ingestion during exercise suppresses exercise-induced cortisol release and attenuates myofibrillar protein degradation.

There are several factors that may determine the effectiveness of CHO-PRO supplementation including the timing and nutritional composition of the supplement. Four studies that supplemented during resistance exercise (Baty et al, 2007 and Bird et al, 2006a, 2006b, 2006c) found reductions in cortisol levels and markers of protein degradation such as 3-methylhistidine and creatine kinase in CHO-PRO groups compared to PLA groups. Conversely, three studies that supplemented after resistance exercise (Williams et al, 2001; Kreider et al, 2007; Rasmussen et al, 2000) found no differences in cortisol levels between the CHO-PRO groups and the PLA groups. The composition of the supplements used ranged from a 6.2\% CHO-1.5\% PRO liquid drink (Baty et al, 2007) to a $40 \mathrm{~g}$ whey protein and $120 \mathrm{~g}$ sucrose shake (Kreider et al, 2007). There is also a difference in the types of $\mathrm{CHO}$ and PRO in each supplement, ranging from $6 \mathrm{~g}$ of essential amino acids to $40 \mathrm{~g}$ of whey protein to $35 \mathrm{~g}$ of sucrose or $120 \mathrm{~g}$ of sucrose. In addition to the composition of the supplements, there is also a difference in the energy content of the CHO-PRO supplements between studies and CHO-PRO, CHO and PLA 
groups within studies. The methods and results of these investigations are summarized in Table 2.

\subsubsection{Effect on Perceived Exertion}

Limited evidence suggests that consumption of a CHO-PRO supplement can attenuate perceived exertion during aerobic endurance exercise (Saunders et al., 2006; Blomstrand et al., 1997), yet it remains unclear whether CHO-PRO can attenuate perceived exertion during resistance exercise. A study by Blomstrand et al. (1997) investigated the effects of a $7 \mathrm{~g} / \mathrm{L}$ PRO supplement (branched-chain amino acids) ingested immediately before and every 15 minutes during exercise on performance and perceived exertion. Subjects exercised on a cycle ergometer for 60 minutes at $70 \%$ of maximal oxygen uptake, followed by another 20 minutes at maximum. Results indicated that subjects' rating of perceived exertion and mental fatigue were lower during exercise when they received PRO supplementation compared to a placebo. It appears that no studies have examined the effect of CHO-PRO supplementation on perceived exertion during resistance exercise.

\subsection{CONCLUSION}

An acute bout of resistance exercise causes significant physiological and metabolic responses that include, but not limited to: 1) decreases in muscle glycogen; 2) increases 
in circulating catabolic hormones such as cortisol; 3) stimulation of protein synthesis and degradation within muscle; and 4) onset of central and peripheral fatigue. Such responses can affect resistance exercise performance, and consequently, adaptations occurring as a result. CHO supplementation during resistance exercise can improve performance by maintaining muscle glycogen stores, suppressing the post-exercise rise in cortisol, increasing the anabolic hormone insulin, and attenuating protein degradation that occurs within a muscle. It appears CHO-PRO supplementation during resistance exercise may be more effective than $\mathrm{CHO}$ supplementation or a PLA for enhancing muscular adaptations and improving recovery. Some evidence suggests CHO-PRO supplementation during resistance exercise will maintain muscle glycogen stores, suppress post-exercise cortisol, increase insulin, attenuate muscle protein degradation, increase muscle protein synthesis, and reduce central fatigue greater than $\mathrm{CHO}$ supplementation or a PLA. Evidence also suggests that CHO-PRO supplementation results in a more anabolic environment for muscular adaptations to occur and improve recovery after exercise. The use of CHO-PRO supplementation during resistance exercise and its effects on volume of work, perceived exertion, and salivary cortisol have not been examined and will be explored in the present investigation. 
Table 2: Summary of CHO-PRO supplementation and resistance exercise.

\begin{tabular}{|c|c|c|c|c|c|}
\hline Article & Subjects & Supplement & Dosage & Protocol & Results \\
\hline $\begin{array}{l}\text { Baty et al, } \\
2007\end{array}$ & $\begin{array}{l}\text {-34 untrained } \\
\text { males (21.5 yrs) }\end{array}$ & $\begin{array}{l}-6.2 \% \text { CHO }-1.5 \% \\
\text { PRO }\end{array}$ & $\begin{array}{l}-355 \mathrm{ml} 30 \mathrm{~min} \\
\text { prior } \\
-177 \mathrm{ml} \\
\text { immediately prior } \\
-177 \mathrm{ml} \text { halfway } \\
-355 \mathrm{ml} \\
\text { immediately post }\end{array}$ & $\begin{array}{l}\text {-single session } \\
-3 \text { sets, } 8 \text { reps } \\
-8 \text { RM to } \\
\text { fatigue }\end{array}$ & $\begin{array}{l}\text {-no difference in } \\
\text { performance } \\
-\uparrow \text { cortisol in PLA } \\
\text { group at } 24 \text { hours } \\
\text { post } \\
-\uparrow \text { insulin in } \\
\text { CHO-PRO group }\end{array}$ \\
\hline $\begin{array}{l}\text { Bird et al, } \\
\text { 2006a }\end{array}$ & $\begin{array}{l}\text {-32 untrained } \\
\text { males (21.0 yrs) }\end{array}$ & $\begin{array}{l}-6 \% \text { CHO } \\
-6 g \text { EAA } \\
-6 \% \text { CHO + 6g } \\
\text { EAA }\end{array}$ & $\begin{array}{l}-8.5 \mathrm{ml} / \mathrm{kg} \text { body } \\
\text { weight }(675 \mathrm{ml}) \\
-22.5 \text { to } 30.0 \mathrm{ml} \\
\text { between each } \\
\text { exercise set }\end{array}$ & $\begin{array}{l}-60 \text { min single } \\
\text { session } \\
-3 \text { sets, } 8-10 \\
\text { reps } \\
-75 \% \text { of } 1 \mathrm{RM}\end{array}$ & $\begin{array}{l}-\uparrow \text { insulin and } \\
\text { glucose in CHO } \\
\text { and CHO-EAA } \\
\text { groups } \\
-\downarrow \text { cortisol in } \\
\text { CHO and CHO- } \\
\text { EAA groups }\end{array}$ \\
\hline $\begin{array}{l}\text { Bird et al, } \\
\text { 2006b }\end{array}$ & $\begin{array}{l}-32 \text { untrained } \\
\text { males (21.0 yrs) }\end{array}$ & $\begin{array}{l}-6 \% \text { CHO } \\
-6 g \text { EAA } \\
-6 \% \text { CHO + 6g } \\
\text { EAA }\end{array}$ & $\begin{array}{l}-8.5 \mathrm{ml} / \mathrm{kg} \text { body } \\
\text { weight }(675 \mathrm{ml}) \\
-22.5 \text { to } 30.0 \mathrm{ml} \\
\text { between each } \\
\text { exercise set }\end{array}$ & $\begin{array}{l}-60 \text { min single } \\
\text { session } \\
-3 \text { sets, } 8-10 \\
\text { reps } \\
-75 \% \text { of } 1 \mathrm{RM}\end{array}$ & $\begin{array}{l}-\uparrow \text { insulin in CHO } \\
\text { and CHO-EAA } \\
\text { groups } \\
-\uparrow \text { cortisol in PLA } \\
\text { group } \\
\text {-No change in } \\
\text { cortisol in CHO } \\
\text { and CHO-EAA } \\
\text { groups }\end{array}$ \\
\hline $\begin{array}{l}\text { Bird et al, } \\
2006 \mathrm{c}\end{array}$ & $\begin{array}{l}\text {-32 untrained } \\
\text { males (21.0 yrs) }\end{array}$ & $\begin{array}{l}-6 \% \text { CHO } \\
-6 \mathrm{~g} \text { EAA } \\
-6 \% \text { CHO + 6g } \\
\text { EAA }\end{array}$ & $\begin{array}{l}-8.5 \mathrm{ml} / \mathrm{kg} \text { body } \\
\text { weight }(675 \mathrm{ml}) \\
-22.5 \text { to } 30.0 \mathrm{ml} \\
\text { between each } \\
\text { exercise set }\end{array}$ & $\begin{array}{l}\text {-same as above } \\
\text { except } \\
-12 \text { weeks } \\
-2 \text { sessions per } \\
\text { week }\end{array}$ & $\begin{array}{l}-\uparrow \text { insulin in CHO } \\
\text { and CHO-EAA } \\
\text { groups } \\
-\downarrow \text { cortisol in } \\
\text { CHO and CHO- } \\
\text { EAA groups }\end{array}$ \\
\hline $\begin{array}{l}\text { Williams et al, } \\
2001\end{array}$ & $\begin{array}{l}-7 \text { resistance } \\
\text { trained males } \\
\text { (21 yrs) }\end{array}$ & $\begin{array}{l}-1.0 \mathrm{~g} / \mathrm{kg} \text { glucose, } \\
.25 \mathrm{~g} / \mathrm{kg} \text { whey } \\
\text { protein, .125 g/kg } \\
\text { leucine and } \\
\text { phenylalanine }\end{array}$ & $\begin{array}{l}-500 \text { ml CHO- } \\
\text { PRO or PLA } \\
\text { immediately after } \\
\text { exercise }\end{array}$ & $\begin{array}{l}\text {-single session } \\
-3 \text { sets, } 10 \text { reps } \\
-100 \% \text { of } 10 \\
\text { RM }\end{array}$ & $\begin{array}{l}-\uparrow \text { insulin in } \\
\text { CHO-PRO group } \\
\text {-no effect on } \\
\text { cortisol }\end{array}$ \\
\hline $\begin{array}{l}\text { Kreider et al, } \\
2007\end{array}$ & $\begin{array}{l}-19 \text { males, } 21 \\
\text { females ( } 23.2 \\
\text { yrs) }\end{array}$ & $\begin{array}{l}-40 \text { g whey protein } \\
-120 \text { g sucrose, } \\
\text { powdered honey, } \\
\text { or maltodexerin }\end{array}$ & $\begin{array}{l}\text {-CHO-PRO } \\
\text { powder in } 16 \mathrm{oz} \\
\text { of water taken } \\
\text { post-exercise }\end{array}$ & $\begin{array}{l}\text {-single session } \\
-3 \text { sets, } 10 \text { reps } \\
-70 \% \text { of } 1 \mathrm{RM}\end{array}$ & $\begin{array}{l}-\uparrow \text { insulin in } \\
\text { CHO-PRO group } \\
\text {-no effect on } \\
\text { cortisol }\end{array}$ \\
\hline $\begin{array}{l}\text { Rasmussen et } \\
\text { al, } 2000\end{array}$ & $\begin{array}{l}-3 \text { male, } 3 \\
\text { female } \\
\text { recreationally } \\
\text { active subjects } \\
\text { (34 yrs) }\end{array}$ & $\begin{array}{l}-6 \text { g EAA } \\
-35 \text { g sucrose }\end{array}$ & $\begin{array}{l}\text {-CHO-PRO } \\
\text { dissolved in } 500 \\
\text { ml water and } \\
\text { taken either } 1 \text { or } 3 \\
\text { hrs post-exercise }\end{array}$ & $\begin{array}{l}-10 \text { sets, } 8 \text { reps } \\
\text { leg press } \\
-8 \text { sets of } 8 \text { reps } \\
\text { leg extension } \\
-80 \% \text { of } 1 \text { RM }\end{array}$ & $\begin{array}{l}-\uparrow \text { insulin in } \\
\text { CHO-PRO group } \\
-\uparrow \text { muscle protein } \\
\text { synthesis }\end{array}$ \\
\hline
\end{tabular}




\subsection{METHODS}

\subsection{EXPERIMENTAL DESIGN}

A single-blind, true experimental design compared the effects of $\mathrm{CHO}-\mathrm{PRO}, \mathrm{CHO}$, and PLA supplementation on RE performance, perceived exertion, and salivary cortisol in a sample of male college aged recreational athletes. Subjects randomly received either a: 1) $\mathrm{CHO}-\mathrm{PRO}$; 2) $\mathrm{CHO}$; or 3) PLA supplement 15 minutes prior to exercise, between every other set during exercise, and immediately post-exercise.

\subsection{SUBJECTS}

Twenty seven college aged (18 to 30 yrs) healthy males who participated in recreational strength training were recruited from the university community to participate in this investigation. Subjects were considered recreationally strength-trained if they performed $\mathrm{RE}$ at least twice but not greater than four times per week over the previous 6 months. To be eligible to participate, subjects were: 1) healthy; 2) non-obese (defined as a BMI <

$30 \mathrm{~kg} / \mathrm{m}^{2}$ ); 3) had a minimum of 6 months resistance training experience; 4) had not participated in collegiate or professional athletics for 6 months prior to the study; and 5) 
willing to undergo all training sessions and familiar with resistance training exercise related to the protocol. Exclusion criteria included: 1) responding yes to one or more questions on PAR-Q; 2) presence of a serious or unstable medical illness; 3) any cardiovascular, musculoskeletal and metabolic contraindications to exercise participation; 4) currently being treated for psychological disorder or having treatment, hospitalization, or emergency room visit in the last 6 months; 5) knowingly taking any performance enhancing substances including anabolic steroids; 6) the use of nutritional supplements of any kind during the 3 weeks prior to the testing; or 7) unwilling to perform or participate in any treatments on testing day. The study received approval by the University of Pittsburgh Institutional Review Board prior to data collection.

\subsection{PROTOCOL}

\subsubsection{Protocol Overview}

Subjects reported to the Human Energy Research Lab for testing on two separate days within a 7 day period of time. On the first day, each subject completed a Physical Activity Readiness Questionnaire (PAR-Q), Medical History Questionnaire, and informed consent prior to entering the study. Subjects then underwent preliminary testing including anthropometric measurements, orientation to OMNI-RES, and determination of maximal strength on a leg press machine. Following the testing, subjects were randomly assigned to one of the three treatment groups. 
On the second day, subjects randomly received either a $6.2 \%$ carbohydrate - 1.7\% protein supplement (CHO-PRO), 7.9\% carbohydrate supplement (CHO) or placebo beverage (PLA) 15 to 30 minutes before the exercise session, between every other set during the exercise session, and immediately post exercise. All subjects completed a RE session consisting of multiple sets of seated leg press exercise starting with 1 set of 10 repetitions at 40,50 , and $60 \%$ of 1 repetition maximum (RM), continuing with multiple sets of 8 to 10 repetitions at $70 \%$ of their 1 RM until fatigue is reached (i.e. unable to complete 8 repetitions). Three minute rest intervals occurred between all sets. Ratings of perceived exertion for the active muscle group (RPE-AM) and overall body (RPE-O) were measured immediately following each set of the seated leg press exercise. Additionally, a rating of perceived exertion for the entire exercise session (S-RPE) was attained 30 minutes after the RE session concluded. Salivary cortisol samples were collected approximately 15 minutes prior to exercise and 30 minutes post-exercise.

\subsubsection{Day 1}

Subjects were instructed to wear loose fitting clothing (i.e. shorts and t-shirt) and to report to the Human Energy Research lab euhydrated and in a 2 hour post-prandial state. In addition, subjects were asked to abstain from their regular exercise routine on testing days. Subjects completed a Physical Activity Readiness Questionnaire (PAR-Q), Medical History Questionnaire, and informed consent. All aspects of study were addressed including the purpose of the study and potential risks and benefits. Each subject was instructed to maintain normal training levels the week between day 1 and day 2 testing sessions. 


\subsubsection{Height, Weight, Body Composition}

Following informed consent, subjects were assessed for height, weight, and body composition. Body height $(\mathrm{cm})$ and weight $(\mathrm{kg})$ were measured using a physician scale (Detecto Scales Inc., Brooklyn, NY). Body composition was determined using a Tanita body fat analyzer (Tanita Corporation of America, Inc. Skokie, IL.). The subject's height, age, and gender were entered into the Tanita analyzer and the "standard" mode for calculating body fat was used for all subjects. Subjects were instructed to remove their shoes and socks and to stand on the Tanita sensors with their bare feet until a body fat reading was determined.

\subsubsection{Orientation to OMNI-RES}

All subjects underwent an OMNI-RES orientation session to ensure familiarity with the scale prior to testing. Both a differentiated (RPE for the active muscle) and an undifferentiated (RPE for the overall body) rating of perceived exertion was assessed during the exercise session. The OMNI-RES scale (Figure 2) was used to assess perceived exertion for the active muscle (RPE-AM) and overall body (RPE-O). Scaling procedures for the OMNI-RES scale were administered to each subject prior to the 1RM test, using the procedures explained by Robertson et al. (2003) (Appendix A). 


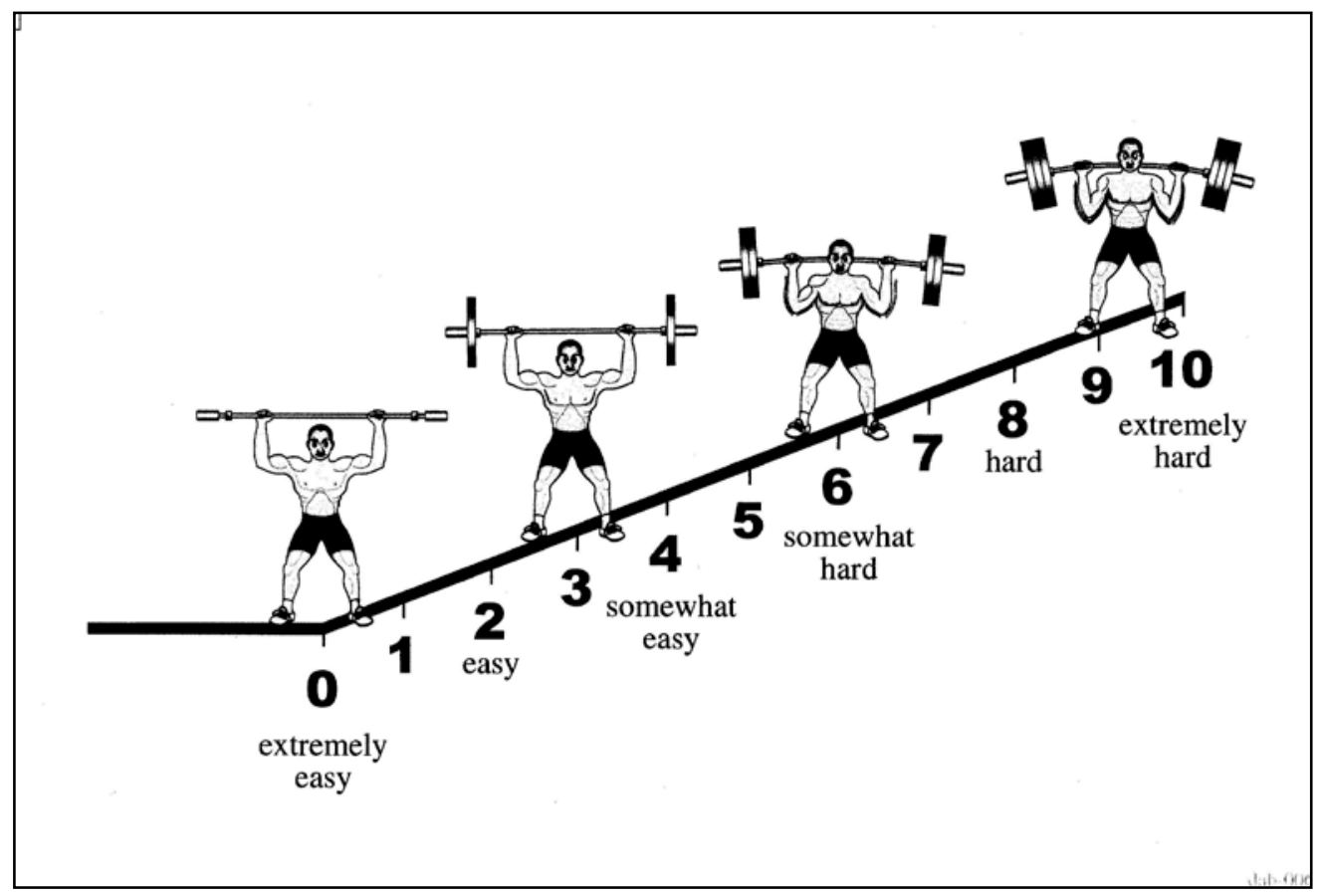

Figure 3: OMNI perceived exertion scale for resistance exercise (OMNI-RES).

\subsubsection{1 Repetition Maximum}

All exercise testing were conducted in the Human Energy Research Lab by a Certified Strength and Conditioning Specialist (CSCS) with the assistance of a trained investigator. 1 RM testing was performed according to a standardized protocol developed by the National Strength and Conditioning Association (NSCA) (Baechle and Earle, 2000). The subject performed an initial warm-up set of 10 repetitions at a self selected light weight on a Cybex leg press machine. Following a 1 minute rest period, weight was increased by $10-20 \%$ for the leg press exercise where 3 to 5 repetitions were performed. Following a 2 minute rest period, a near-maximum load was estimated by the trained investigator and performed by the subject for up to 3 repetitions. Following a 3 minute rest period, the load was again increased by $10-20 \%$. The subject then attempted a 
1RM. If successful, the subject rested for 3 minutes and attempted a weight increased by the same methods as previously stated. Once the subject was unable to lift a weight, the highest weight lifted successfully was the 1RM. Ideally, this was measured within five testing sets in order to prevent performance decrements due to fatigue.

\subsubsection{Diet Record}

For the 7 days prior to the day 2 experimental trial, subjects were encouraged to maintain their current dietary habits and avoid taking nutritional supplements of any kind. Subjects were instructed to record their food intake for the 3 days prior to the experimental trial in a food journal provided to them by the investigator. Subject diets were analyzed in order to determine any inconsistencies in macronutrient intake between groups that may affect the outcome of the experimental trial. The three day diet records were analyzed using a computerized dietary assessment program (FitDay.com, Internet Brands Inc.) by a trained investigator. 


\subsubsection{Day 2}

\subsubsection{Supplementation Protocol}

Approximately 7 days following the initial assessment, subjects reported to the Human Energy Research Lab between the hours of 8:00 and 10:00 am having fasted overnight for 12 hours. All testing took place at the same time of day to control for variations in cortisol levels. Subjects were provided a standardized liquid meal which served as a control for any dietary differences that may have existed among the subjects. The standardized liquid meal contained: 1) 50\% of energy as carbohydrate; 2) $34 \%$ as fat; and 3)16\% as protein (Boost Plus, Mead Johnson Nutritionals, Evansville, IN) and was ingested at an energy level of $12 \mathrm{kcal} \cdot \mathrm{kg}^{-1}$ of body mass. Subjects reported back to the lab between 1:00 and 3:00 pm not having ingested energy in any form for 5 hours. Subjects were also instructed to not chew gum or brush their teeth for 30 minutes before reporting back to the lab and were seated for 15 minutes prior to the collection of the resting salivary cortisol sample.

After the salivary cortisol sample was collected (see section 3.3.4), subjects received either a $6.2 \%$ carbohydrate $-1.7 \%$ protein supplement (CHO-PRO), $7.9 \%$ carbohydrate supplement (CHO) or PLA 15 minutes prior to the start of the exercise session $\left(8 \mathrm{ml} \cdot \mathrm{kg}^{-1}\right)$ and immediately post-exercise at a rate of $\left(8 \mathrm{ml} \cdot \mathrm{kg}^{-1} \cdot \mathrm{hr}\right)$. Subjects also received supplementation between every other set (approximately every $7.5 \mathrm{~min}$ ) throughout the exercise session $\left(8 \mathrm{ml} \cdot \mathrm{kg}^{-1}\right.$ divided into 8 supplements per hour). The CHO-PRO and CHO supplements consisted of equal fluid volumes and were 
approximately isocaloric (Table 3), controlling for differences in energy content between the supplements that may affect the outcome. In order to match the caloric content of the supplements, the $\mathrm{CHO}$ supplement was mixed at a higher concentration than company recommendations. The carbohydrate in the CHO supplement consisted of sucrose and dextrose, while the carbohydrate in the CHO-PRO supplement consisted of sucrose and fructose. The protein in the CHO-PRO supplement consisted of whey protein isolate and contained branched-chain amino acids (leucine, isoleucine and valine). All three supplements were similar in appearance and taste. All supplementation for a given subject was prepared and ready prior to the experimental trial.

Table 3: Supplementation Beverage Formulation

\begin{tabular}{c|c|c|c}
\hline & Calories per Serving* & CHO per Serving* & PRO per Serving* \\
\hline CHO-PRO & 80 & $15 \mathrm{~g}$ & $4 \mathrm{~g}$ \\
CHO & 75 & $19 \mathrm{~g}$ & $0 \mathrm{~g}$ \\
\hline
\end{tabular}

* 1 serving $=8 \mathrm{fl.oz.} \mathrm{or} 240 \mathrm{ml}$

\subsubsection{Resistance Exercise Protocol}

The exercise protocol consisted of performing multiple sets of seated leg press exercise until fatigue was reached (Figure 3). Fatigue was considered the point at which a subject could not complete the required $8-10$ repetitions or could no longer continue due to exhaustion. Subjects began with a warm-up consisting of 5 minutes of riding a Monark Cycle Ergometer (Monark Exercise Ab, Vansbro, Sweden) with low resistance (0.5 kg) at 50 rpm followed by standardized lower body static stretching supervised by a trained 
investigator. A warm-up set followed where subjects performed one set of 10 repetitions at $40 \%$ of their $1 \mathrm{RM}$ as determined by a trained investigator. Each repetition had a cadence of 2 seconds for the concentric movement and 2 seconds for the eccentric movement with the aid of a metronome (ACSM Position Stand, 2002). For the subsequent 2 warm-up sets, subjects performed 1 set of 10 repetitions at both 50 and $60 \%$ of $1 \mathrm{RM}$.

Following the warm-up, subjects performed multiple sets of 8 to 10 repetitions at 70\% of their $1 \mathrm{RM}$ until fatigue. A 3 minute rest period, timed by a trained investigator, followed each set. Supplementation occurred during the rest period after every other set. Ratings of perceived exertion for the active muscle (RPE-AM) and overall body (RPE-O) were obtained immediately after each set of seated leg press exercise using the OMNIRES scale. The OMNI-RES scale was positioned in clear view for subjects to see during the entire session. A rating of perceived exertion for the entire exercise session (S-RPE) was obtained with the subjects in a seated position 30 minutes following the resistance exercise session.

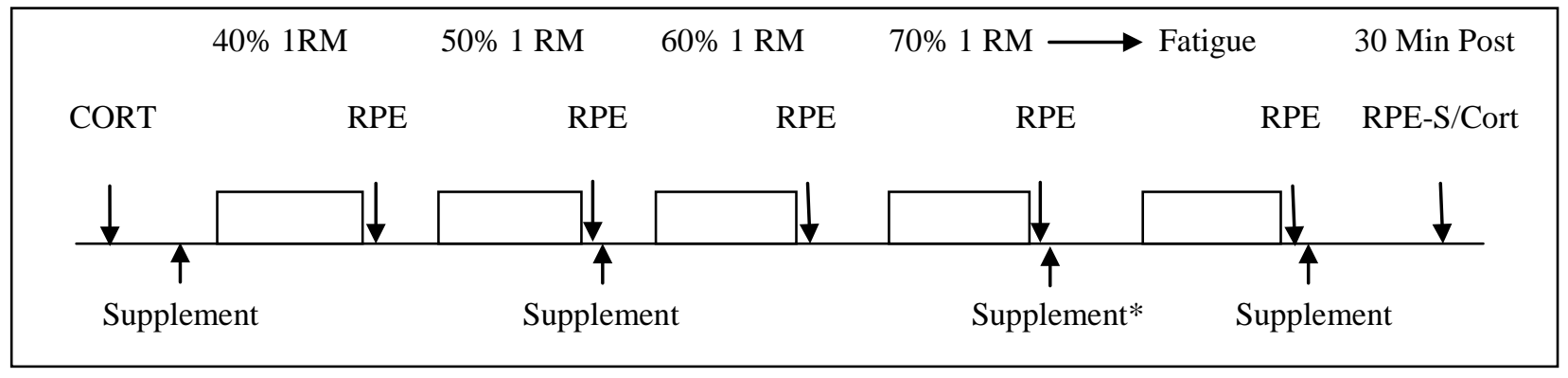

Figure 4: Resistance exercise protocol and measurement time points.

* Supplementation will occur between every other set until fatigue is reached. 


\subsubsection{Measurement of Salivary Cortisol}

Salivary cortisol samples were collected immediately before exercise (PRE) and 30 min post-exercise (POST). For each measurement, saliva was deposited into sterile containers by the subject with the aid of a straw until the container was half full. All samples were labeled and refrigerated until sent to the testing laboratory to be analyzed. At the completion of exercise, subjects remained seated in the laboratory for 30 min before the post-exercise sample were collected in order to avoid contamination of salivary cortisol sample from the supplementation. Salivary samples were analyzed by Salimetrics, LLC (State College, PA) and were reported in nmol/L.

\subsection{VARIABLES}

The independent variable in this study was the type of supplement consumed (CHOPRO, CHO, or PLA) prior to, during, and following RE. Dependent variables in this study included: 1 ) volume of work output (weight $(\mathrm{kg}) \times$ sets $\times$ repetitions); 2) salivary cortisol responses (nmol/L); 3) ratings of perceived exertion for the active muscle (RPEAM); 4) overall body (RPE-O); and 5) entire exercise session (S-RPE) measured with the OMNI Perceived Exertion Scale for Resistance Exercise (OMNI-RES). Dependent measures of performance and perceived exertion were conducted prior to, during, and following the treatment supplementation. 


\subsection{STATISTICAL ANALYSIS}

Data analysis was performed using SPSS 16.0 for Windows statistical software. Descriptive data for subject characteristics and dependent variables were expressed as mean \pm standard deviation. Using a statistical power of 0.80 , and an effect size of 0.50 , it was determined that a sample size of 45 subjects (15 per treatment group) be used in order to test for a main effect. Initial assessments of salivary cortisol and 1RM strength were examined with a one-factor (Treatment) between subjects analysis of variance (ANOVA) to detect group differences. If significant differences occurred in 1RM strength, analyses of covariance were conducted with strength as a covariate.

Volume of work output and session RPE were examined with a one-factor (Treatment) between subjects ANOVA. Ratings of perceived exertion at 25, 50, 75, and $100 \%$ of the total volume of work and salivary cortisol responses were examined with a two-factor (Time x Treatment) mixed ANOVA. A Tukey post-hoc analysis was conducted to detect significance in the treatment factor. Statistical significance was accepted at the $\mathrm{p}<0.05$ level of confidence. 


\subsection{RESULTS}

The purpose of this investigation was to determine the effect of carbohydrate-protein (CHO-PRO) versus carbohydrate (CHO) supplementation and a placebo (PLA) on RE performance, perceived exertion during RE, and post-exercise salivary cortisol in a sample of male college aged recreational athletes. It was hypothesized that CHO-PRO supplementation would increase performance during RE, reflected by volume of work output (sets $\times$ repetitions $\times$ weight), when compared to $\mathrm{CHO}$ supplementation or the PLA condition.

It was also hypothesized that CHO-PRO supplementation would result in a comparatively greater attenuation of perceived exertion during RE for the active muscle (RPE-AM), overall body (RPE-O), and for the entire exercise session compared to $\mathrm{CHO}$ supplementation or the PLA condition. Finally, it was hypothesized that CHO-PRO supplementation would result in a reduced pre-exercise to post-exercise salivary cortisol response when compared to CHO supplementation or the PLA condition.

\subsection{SUBJECT CHARACTERISTICS}

Twenty seven healthy recreationally trained males aged $22.7 \pm 2.3$ years were recruited from the university community to participate in this investigation. One subject was 
excluded from data analysis due to improper $1 \mathrm{RM}$ measurement resulting in an abnormal performance during the day 2 resistance exercise protocol. Another subject was excluded from the investigation after failing to attend the day 2 resistance exercise session.

Subject descriptive characteristics for the three treatment groups and overall sample are included in Table 4. A one-factor between subjects analysis of variance revealed no significant differences between treatment groups for baseline measures of strength (1-RM) or resting salivary cortisol.

Table 4: Subject Descriptive Characteristics.

\begin{tabular}{l|c|c|c}
\hline & CHO (n = 9) & CHO-PRO (n = 9) & PLA (n = 9) \\
\hline \hline Age (yr) & $22.3 \pm 2.6$ & $22.6 \pm 1.5$ & $23.0 \pm 2.6$ \\
Height (cm) & $173.4 \pm 5.8$ & $177.7 \pm 6.5$ & $177.7 \pm 7.4$ \\
Weight (kg) & $72.8 \pm 7.3$ & $80.4 \pm 13.4$ & $78.9 \pm 9.3$ \\
\% Body Fat & $17.1 \pm 4.6$ & $17.9 \pm 7.4$ & $17.9 \pm 3.3$ \\
LBM (kg) & $60.1 \pm 4.8$ & $65.2 \pm 7.2$ & $64.7 \pm 6.5$ \\
$\mathbf{1}$ RM (kg) & $294.9 \pm 100.6$ & $278.3 \pm 59.8$ & $281.3 \pm 48.1$ \\
$\mathbf{1}$ RM $\cdot \mathbf{~ k g ~}^{-1} \mathbf{L B M}^{-1}$ & $4.9 \pm 1.5$ & $4.2 \pm 0.7$ & $4.4 \pm 0.8$ \\
Rest Cort (nmol·L & $0.16 \pm 0.03$ & $0.20 \pm 0.12$ & $0.23 \pm 0.12$ \\
\hline
\end{tabular}

\section{Data are mean \pm standard deviation.}

Analysis of 3-day diet records prior to the treatment protocol were used to determine dietary energy intake and macronutrient composition. A one-factor between subjects analysis of variance performed on daily energy intake and macronutrient 
composition between the three treatment groups revealed no significant difference in daily calorie intake $(p=0.860)$. In addition, no significant differences in carbohydrate intake ( $p=0.220)$, protein intake $(p=0.623)$, or fat intake $(p=0.188)$ occurred between the treatment groups. A summary of daily energy intake and macronutrient composition is presented in Table 5 .

Table 5: Dietary Energy Intake and Macronutrient Composition.

\begin{tabular}{l|c|c|c}
\hline & CHO (n = 9) & CHO-PRO (n = 9) & PLA (n = 9) \\
\hline \hline Carbohydrate Intake (\%) & $43.2 \pm 3.5$ & $51.7 \pm 14.1$ & $44.0 \pm 5.1$ \\
Protein Intake (\%) & $18.3 \pm 8.5$ & $16.8 \pm 4.4$ & $20.2 \pm 3.3$ \\
Fat Intake (\%) & $32.5 \pm 8.4$ & $26.3 \pm 7.6$ & $33.5 \pm 4.1$ \\
Total Calories (kcal·day $\left.{ }^{-1}\right)$ & $2532 \pm 596$ & $2493 \pm 899$ & $2319 \pm 596$ \\
\hline
\end{tabular}

Data are mean \pm standard deviation.

Subjects received either a $6.2 \%$ carbohydrate - $1.7 \%$ protein supplement (CHOPRO), 7.9\% carbohydrate supplement (CHO) or PLA 15 minutes prior to the start of the exercise session $\left(8 \mathrm{ml} \cdot \mathrm{kg}^{-1}\right)$, between every other set (approximately every $7.5 \mathrm{~min}$ ) throughout the exercise session $\left(8 \mathrm{ml} \cdot \mathrm{kg}^{-1}\right.$ divided into 8 supplements per hour), and immediately post-exercise at a rate of $\left(8 \mathrm{ml} \cdot \mathrm{kg}^{-1} \cdot \mathrm{hr}\right)$. The total supplement intake for the three treatment groups is summarized in Table 6 . 
Table 6: Total Supplement Intake Before and During RE Session.

\begin{tabular}{l|c|c|c}
\hline & CHO (n = 9) & CHO-PRO (n = 9) & PLA (n = 9) \\
\hline \hline Total Supplement (ml) & $1122.7 \pm 268.5$ & $1259.0 \pm 275.1$ & $1047.8 \pm 99.0$ \\
Total Calories (kcal) & $350.8 \pm 83.9$ & $419.7 \pm 91.7$ & $21.8 \pm 2.1$ \\
Total Carbohydrate (g) & $88.9 \pm 21.3$ & $78.7 \pm 17.2$ & $0.0 \pm 0.0$ \\
Total Protein (g) & $0.0 \pm 0.0$ & $20.9 \pm 4.6$ & $0.0 \pm 0.0$ \\
\hline
\end{tabular}

\section{Data are mean \pm standard deviation.}

\subsection{RESISTANCE EXERCISE PERFORMANCE}

A one-factor between subjects ANOVA was used to determine the effects of nutritional supplementation on resistance exercise performance. Resistance exercise performance was identified by the total number of repetitions completed and volume of work performed (sets $\times$ repetitions $\times$ weight). The CHO, CHO-PRO, and PLA groups performed an average of $135.6 \pm 54.6,136.3 \pm 36.2$, and $89.9 \pm 15.3$ repetitions, respectively (Figure 5). Results indicated a significant differences in total number of repetitions performed between the three treatment groups $(p=0.027)$. Post-hoc analysis revealed the PLA group was significantly less than the CHO group $(p=0.050)$ and CHOPRO group $(p=0.046)$. There was no significant difference between the CHO and CHOPRO groups $(p=0.999)$. 


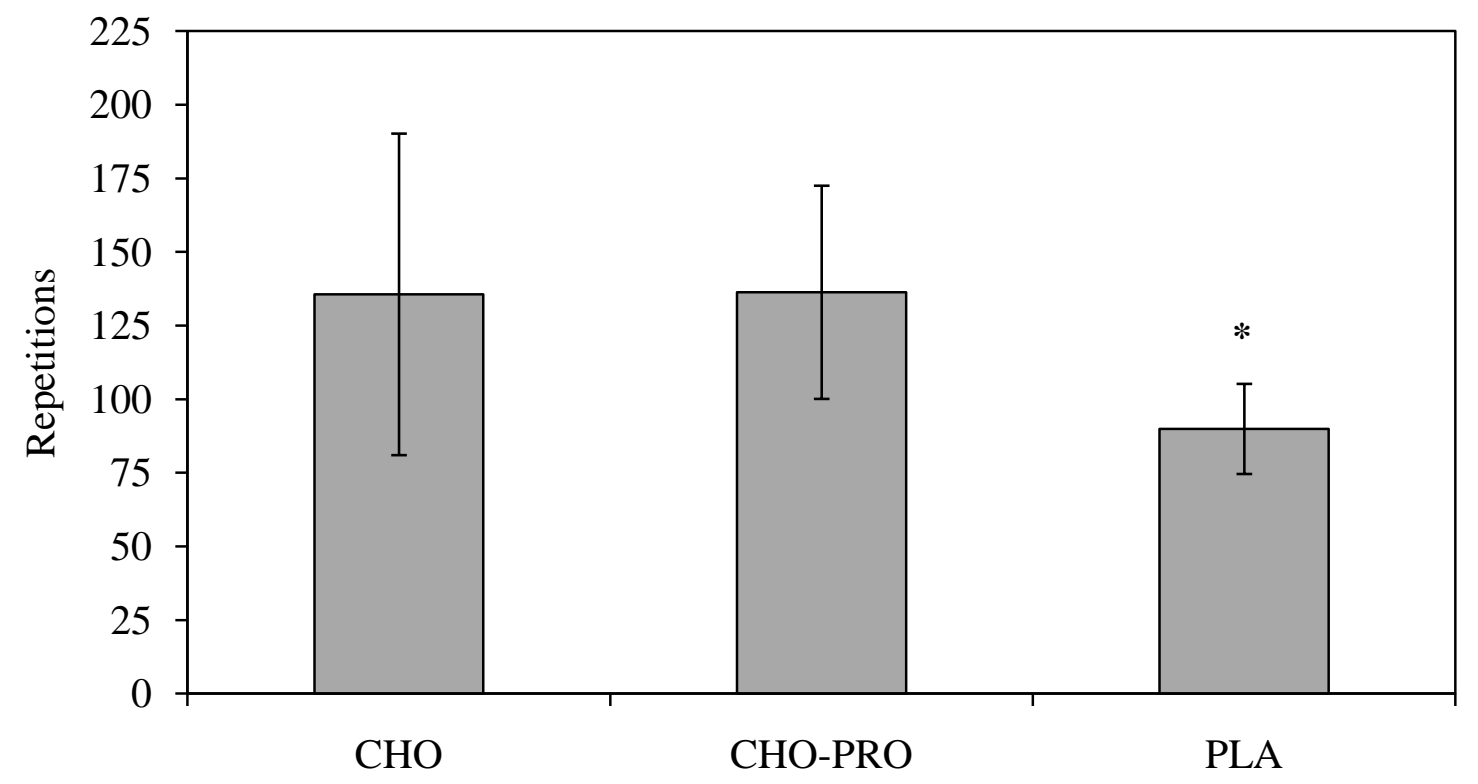

Figure 5: Total Repetitions Performed Following Supplementation and Training Protocol.

* Significantly less than CHO and CHO-PRO groups ( $<<0.05)$.

The average total volume of work performed per group was 28,052 \pm 19,198 kg (CHO), 24,836 \pm 9,737 kg (CHO-PRO), and 15,934 \pm 3,276 kg (PLA) (Figure 6). There was no significant difference in total volume of work performed between the three treatment groups $(p=0.128)$. Additionally, there was no difference in total volume of work performed between groups when expressed relative to lean body mass $(p=.075)$ (Figure 7). 


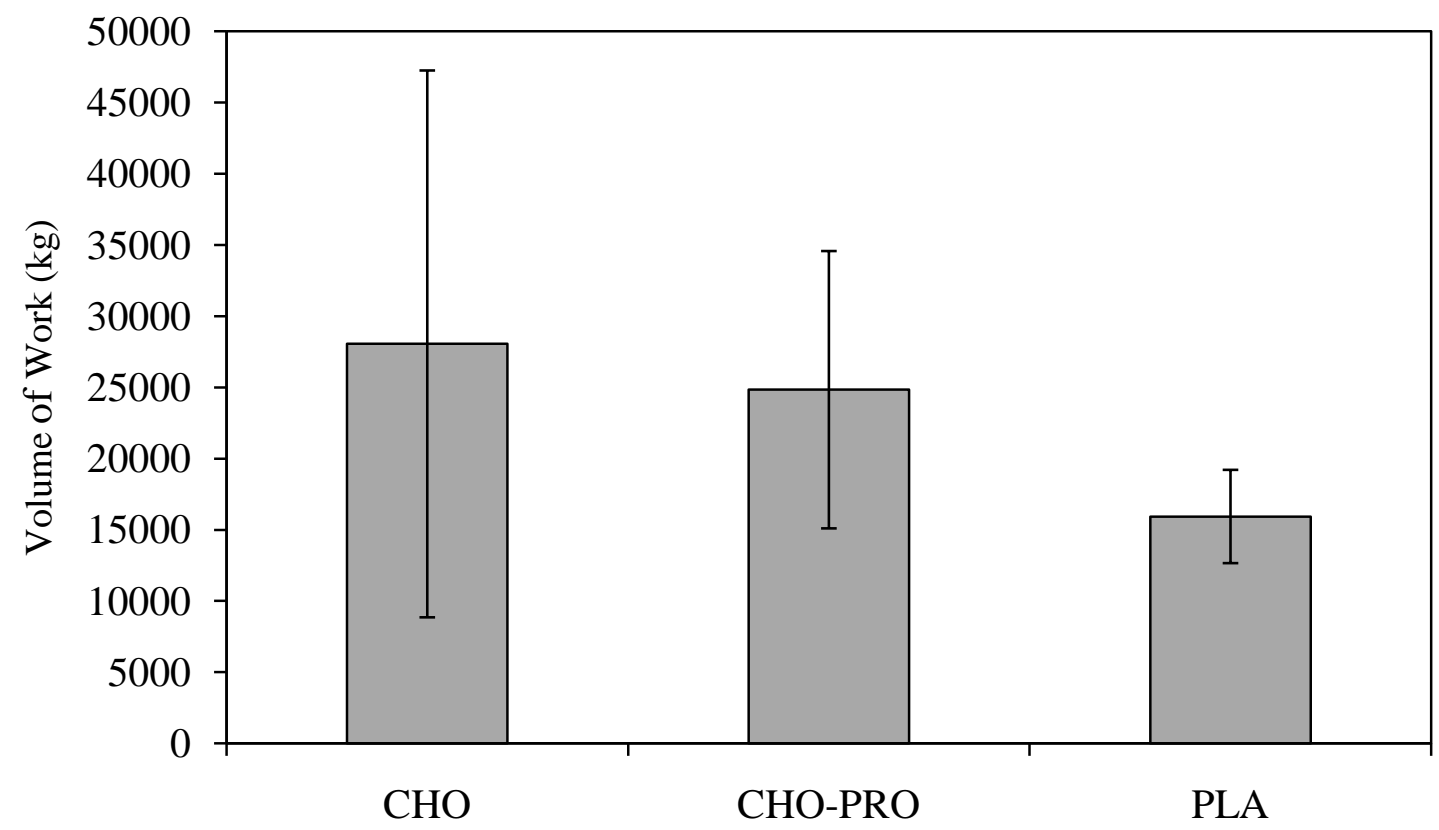

Figure 6: Total Volume of Work Performed Following Supplementation and Training Protocol.

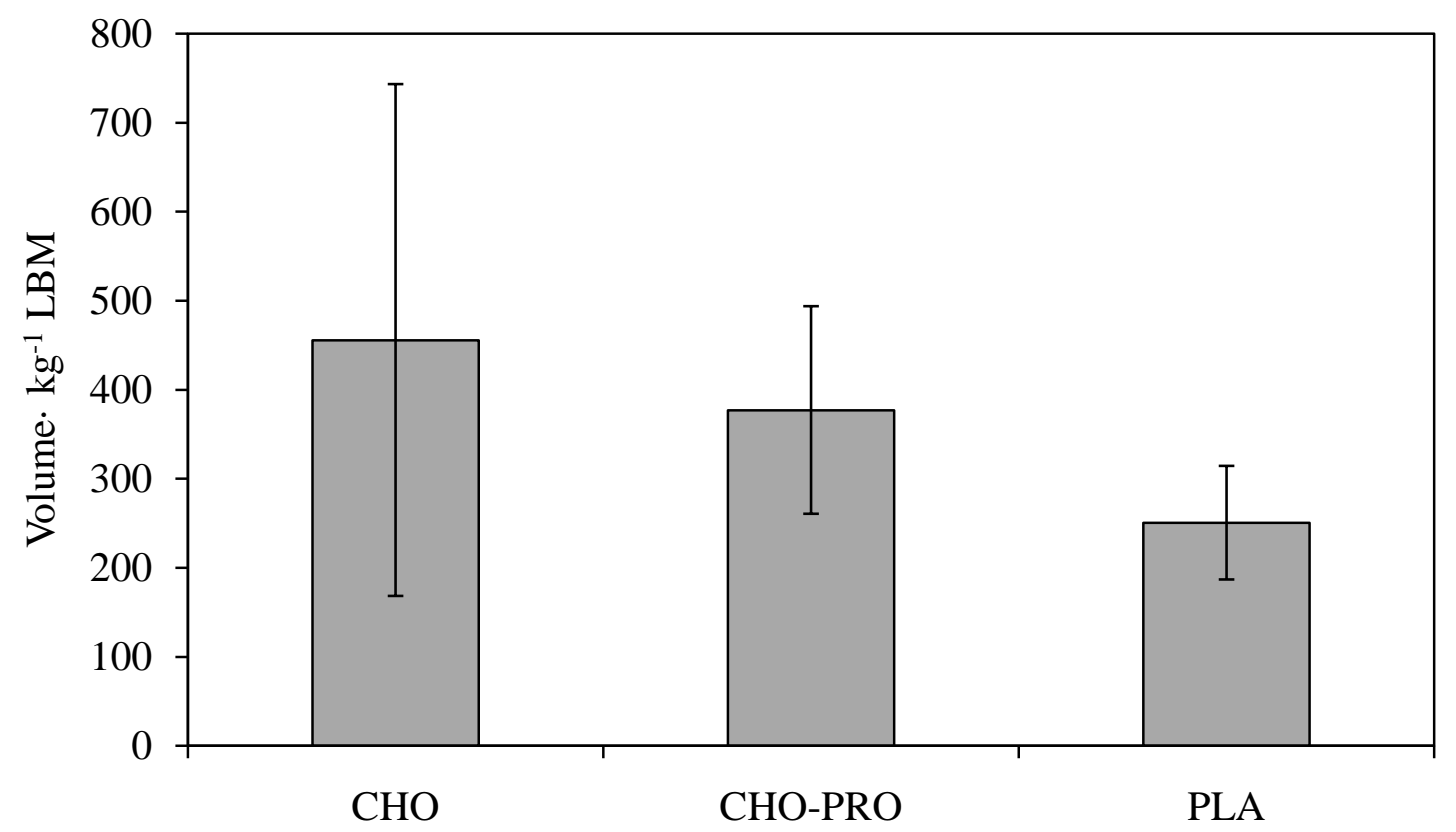

Figure 7: Relative Volume of Work (LBM) Following Supplementation and Training Protocol. 


\subsection{PERCEIVED EXERTION}

A two-factor (group $\times$ time) ANOVA was used to examine ratings of perceived exertion at 25,50 , and $75 \%$ of the total volume of work performed throughout the treatment protocol. Perceived exertion was estimated for active muscle (RPE-AM) and overall body (RPE-O) following each leg press set performed throughout the treatment protocol. A significant time effect was observed for both RPE-AM and RPE-O across time points corresponding to $25,50,75$, and $100 \%$ volume of work $(p<0.001)$. A significant difference in RPE-AM occurred between the three treatment groups at 25\% $(p=0.030)$ and $50 \%$ of total volume $(p=0.031)$. No significant differences were observed between the treatment groups at $75 \%$ of total volume $(p=0.100)$ (Figure 8).

RPE-AM

Post-hoc analyses revealed a significant difference in RPE-AM between the CHO and CHO-PRO groups at $25 \%$ total volume $(p=0.032)$ and $50 \%$ total volume $(p=$ 0.036). There were no significant differences observed between the CHO and PLA groups or CHO-PRO and PLA groups at $25 \%$ or $50 \%$ of total volume $(p>0.05)$ (Figure 8).

$R P E-O$

There were no significant differences between treatment groups for RPE-O at $25 \%$ of total volume $(p=0.072)$, $50 \%$ of total volume $(p=0.173)$, or $75 \%$ of total volume of work $(p=0.360)$ (Figure 9). 


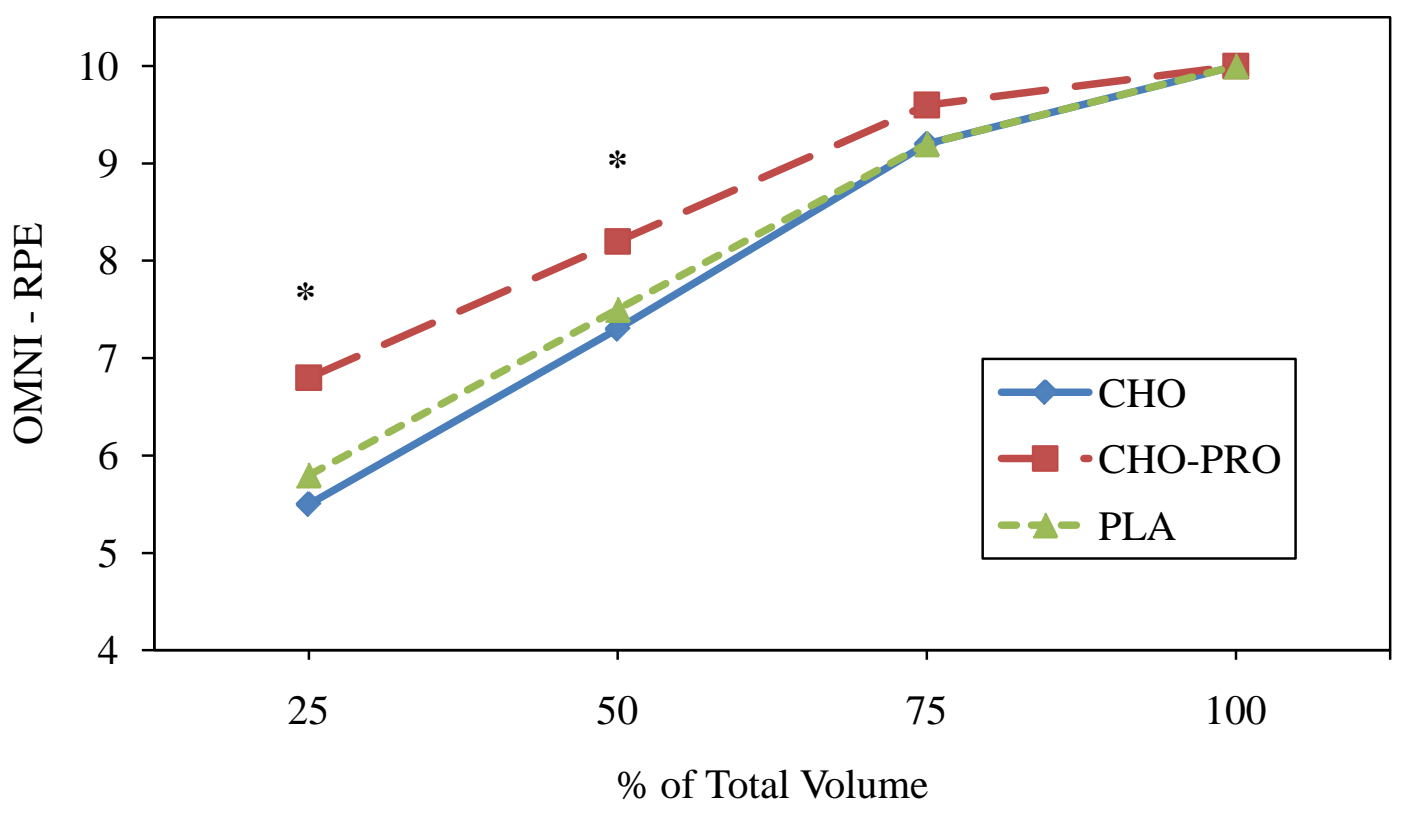

Figure 8: RPE-AM at \% of Total Volume of Work Performed.

* Significant difference from CHO group $(p<0.05)$.

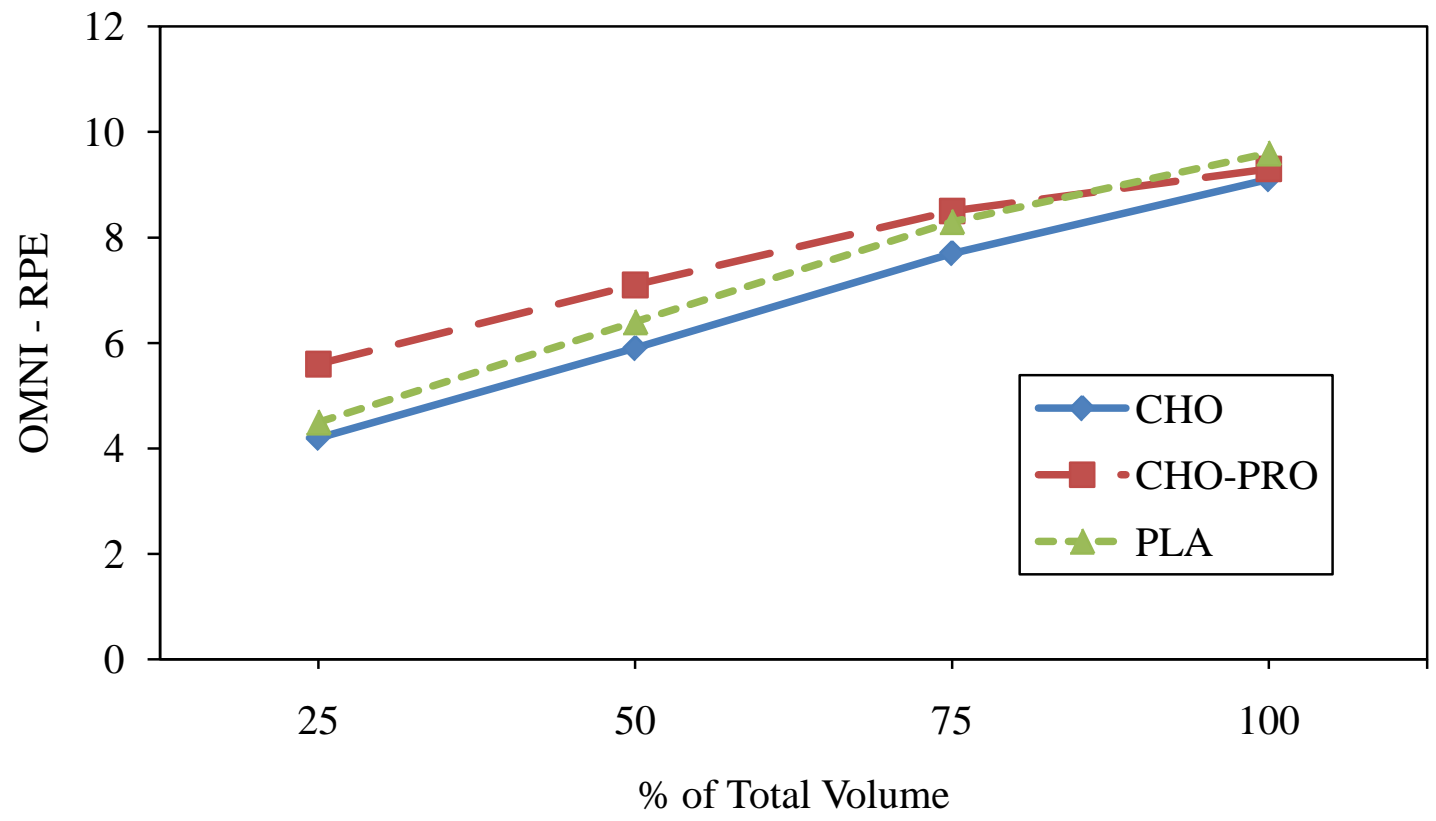

Figure 9: RPE-O at \% of Total Volume of Work Performed. 


\section{Session RPE}

A one-factor between subjects ANOVA was used to determine the effects of nutritional supplementation during resistance exercise on session ratings of perceived exertion. A session rating of perceived exertion for both active muscle (Session RPEAM) and overall body (Session RPE-O) was measured 30 minutes post-exercise. The average ratings of perceived exertion for RPE-AM were $7.7 \pm 0.9,8.6 \pm 1.0$, and $8.2 \pm$ 1.0 for the CHO, CHO-PRO, and PLA groups (Figure 10). No significant difference in Session RPE-AM occurred between the three treatment groups ( $p=0.157)$. Also, the CHO, CHO-PRO, and PLA groups gave an average rating of $5.4 \pm 1.4,6.2 \pm 1.2$, and 6.4 \pm 1.0 , respectively for overall body (Figure 11). There was no significant difference in Session RPE-O between the three treatment groups $(p=0.212)$.

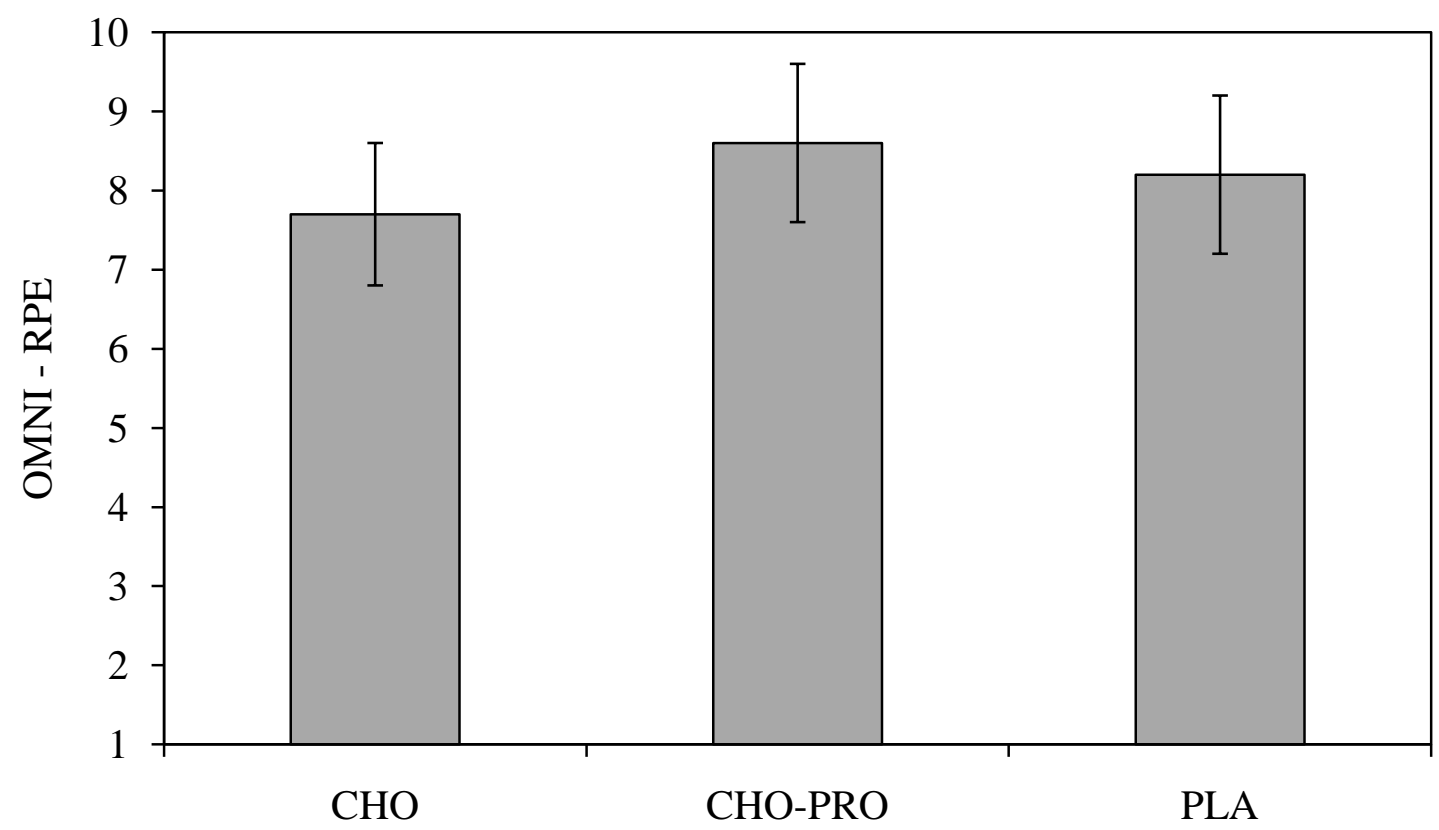

Figure 10: Session RPE-AM 


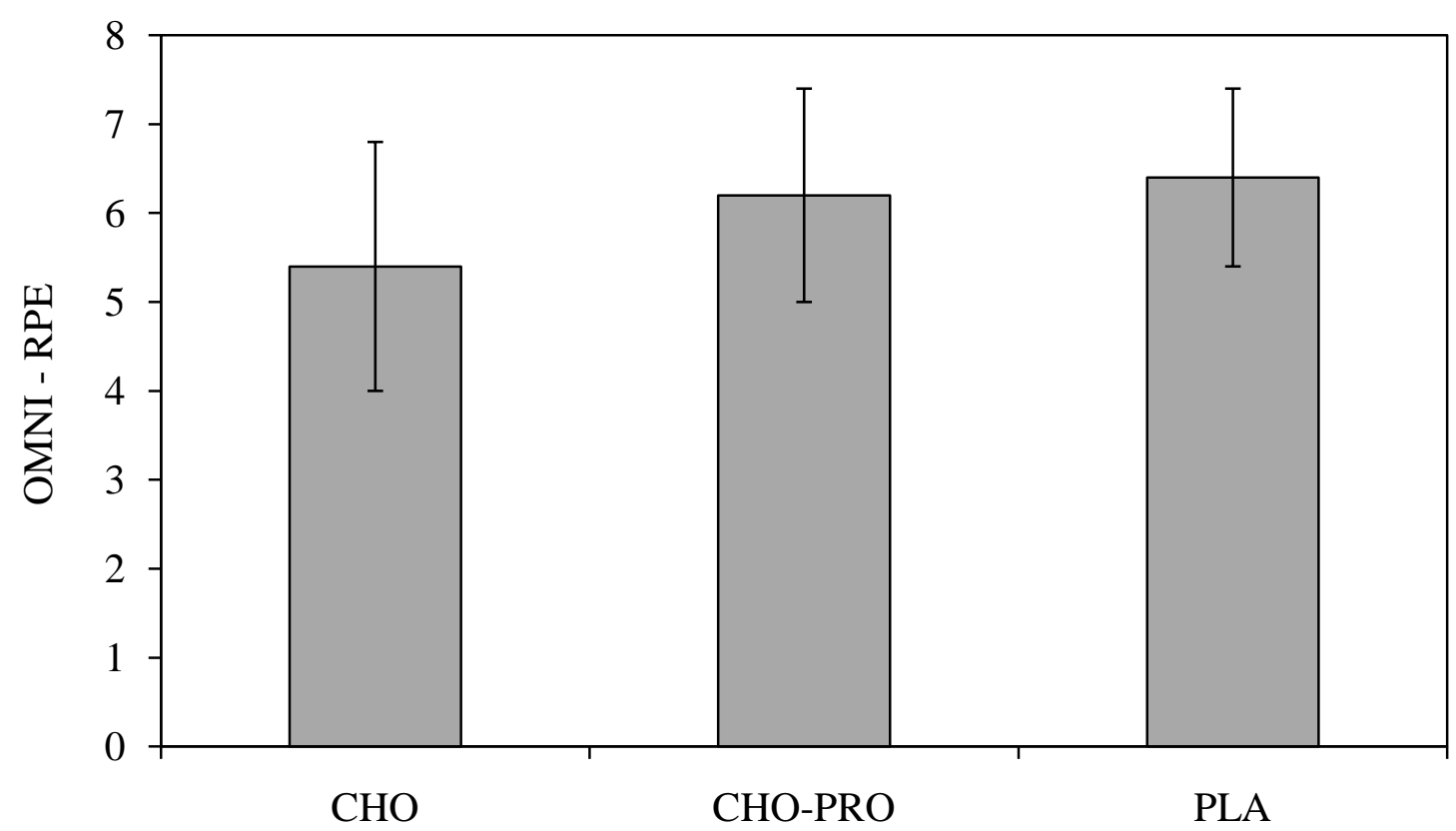

Figure 11: Session RPE-O

\subsection{SALIVARY CORTISOL}

A two-factor (group $\times$ time) ANOVA was used to determine differences in pre-exercise and 30 minutes post-exercise salivary cortisol measurements between the three treatment groups. Results showed no significant interaction group $\times$ time effect between pre and post-exercise salivary cortisol for the CHO, CHO-PRO, and PLA groups $(p>0.05)$ (Figure 12). There was a significant group effect reflecting a difference in post-exercise salivary cortisol between the three treatments $(p=0.035)$. Post-hoc analysis revealed a significant difference in post-exercise salivary cortisol between the CHO and PLA groups $(p=0.039)$ only . 


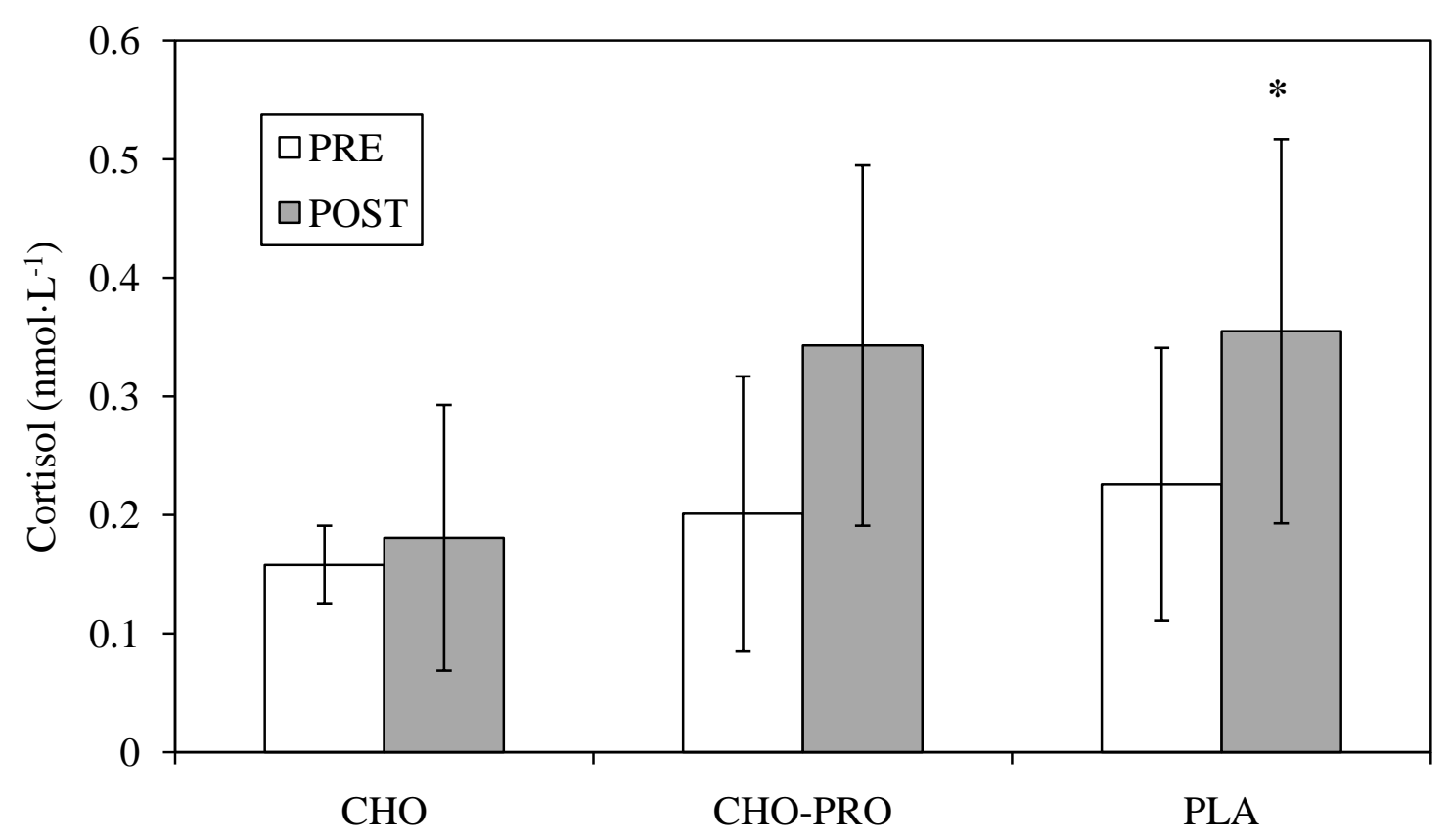

Figure 12: Salivary Cortisol Responses

* Significant difference from CHO group $(\mathrm{p}<0.05)$

\subsection{SUMMARY}

This investigation demonstrated that nutritional supplementation can affect moderateintensity, high-volume resistance exercise performance. CHO-PRO and $\mathrm{CHO}$ supplementation resulted in a significantly greater total number of repetitions performed to fatigue than a PLA. However, the effects of supplementation on perceived exertion appeared to be inconsistent. CHO supplementation resulted in an attenuated RPE-AM response at $25 \%$ and $50 \%$ of total volume of work performed compared to the CHO-PRO supplementation or a PLA. There were no significant differences for RPE-O or SessionRPE between the three treatment groups. Finally, this investigation demonstrated that 
CHO supplementation before and during moderate-intensity, high-volume resistance exercise resulted in an attenuated post-exercise salivary cortisol response compared to a PLA. 


\subsection{DISCUSSION}

The purpose of this investigation was to determine the effect of carbohydrate-protein (CHO-PRO) versus carbohydrate (CHO) supplementation and a placebo (PLA) on RE performance, perceived exertion during RE, and post-exercise salivary cortisol in a sample of male college aged recreational athletes. It was hypothesized that CHO-PRO supplementation would increase performance (reflected by volume of work output [sets $\times$ repetitions $\times$ weight]) during $\mathrm{RE}$ when compared to $\mathrm{CHO}$ supplementation or the PLA condition. Results indicated no significant differences in the total volume of work performed between the three treatment groups. However, the $\mathrm{CHO}-\mathrm{PRO}$ and $\mathrm{CHO}$ groups performed a significantly greater total number of repetitions than the PLA group.

It was also hypothesized that CHO-PRO supplementation would result in a comparatively greater attenuation of perceived exertion during RE for the active muscle (RPE-AM), overall body (RPE-O), and for the entire exercise session compared to CHO supplementation or the PLA condition. Results revealed a significantly lower RPE-A for the CHO group compared to the CHO-PRO group at $25 \%$ and $50 \%$ of total volume of work performed. There were no significant differences for RPE-O or Session RPE.

Finally, it was hypothesized that CHO-PRO supplementation would result in a reduced pre-exercise to post-exercise salivary cortisol response when compared to $\mathrm{CHO}$ supplementation or the PLA condition. On the contrary, there were no differences 
between the CHO-PRO and CHO groups, and a significantly lower post-exercise salivary cortisol response occurred for the CHO group compared to the PLA group.

\subsection{RESISTANCE EXERCISE PERFORMANCE}

Previous research has shown that muscle glycogen stores can decrease by up to $40 \%$ during moderate intensity, high volume RE (Haff et al., 2000; MacDougall et al., 1999;

Pascoe et al., 1993; Robergs et al., 1991; Tesch et al., 1996, 1998) resulting in increased muscular weakness, decreased maximal muscular strength, and decreased force production (Yaskpelkis et al., 1993; Jacobs et al., 1981; Hepburn et al., 1982). Consuming a CHO supplement during RE may help to maintain muscle glycogen stores and improve performance. The addition of protein to a CHO supplement has been shown to increase insulin response and improve maintenance of muscle glycogen stores when compared to CHO supplementation alone (Ivy et al., 2003; Van Loon et al., 2000; Zawadzki et al., 1992). Evidence has also indicated that CHO-PRO supplementation results in a similar or greater improvement in performance compared to $\mathrm{CHO}$ supplementation during aerobic endurance exercise (Saunders et al., 2004; Ivy et al., 2003; Romano et al., 2006). However, it remains unclear whether CHO-PRO supplementation can improve performance during RE. Therefore, the main purpose of this investigation was to determine the effect CHO-PRO supplementation versus $\mathrm{CHO}$ supplementation and a PLA on resistance exercise performance. 
In the present study, it was hypothesized that CHO-PRO supplementation would increase performance during RE when compared to $\mathrm{CHO}$ supplementation or the PLA condition. Results indicated CHO-PRO and CHO supplementation had similar increases in number of repetitions to fatigue compared to the PLA group. This is in contrast to previous work by Ivy et al. (2003) and Saunders et al. (2004) who found CHO-PRO supplementation to be more effective than $\mathrm{CHO}$ supplementation during aerobic endurance exercise. A possible explanation of why results between the current and previous studies differed involves the use of "isocaloric" supplements. Previous studies comparing CHO-PRO to $\mathrm{CHO}$ supplementation have not accounted for higher caloric content of CHO-PRO supplements (Ivy et al., 2003; Saunders et al., 2004). In contrast, the present investigation did administer an "isocaloric" supplementation protocol. While the different supplements may have a similar amount of carbohydrate (6 to 8\%), the extra calories per volume from a CHO-PRO supplement can be attributed to the addition of protein. The protein can be converted to glucose via gluconeogenesis in the liver and provide more useable energy per volume consumed than a $\mathrm{CHO}$ only supplement. Similar to the present experimental design, Romano et al. (2006) did account for differences in caloric content between CHO-PRO and CHO supplements and found no significant difference in aerobic endurance exercise performance between groups.

Similarly, the CHO-PRO and CHO groups both demonstrated a non-significant increased total volume of work performed to fatigue during RE compared to the PLA group. Previous research has been inconsistent with regards to the effectiveness of CHOPRO and CHO supplementation and their ability to maintain muscle glycogen and improve performance during resistance exercise (Kulick et al., 2008; Baty et al., 2007; 
Haff et al., 2001, Haff et al., 1999, and Lambert et al., 1991). Baty et al. (2007) found CHO-PRO supplementation did not improve RE performance. Haff et al. (2001) found CHO supplementation did improve performance during RE, while Kulik et al (2008) found CHO supplementation did not improve RE performance. The inconsistencies in results may be due to the various RE protocols used in the investigations.

Haff et al. (2003) suggests in order for supplementation to improve resistance exercise performance, a protocol needs to exceed 50 minutes in duration and focus on one major muscle group in order to sufficiently deplete muscle glycogen stores. In the study by Baty et al. (2007) the RE protocol exceeded 50 minutes in duration but utilized total body exercises instead of focusing on a single major muscle group. In contrast, the RE protocol used by Kulick et al. (2008) was below the recommended 50 minutes in duration (approximately $30 \mathrm{~min}$ ) but did focus on one major muscle group (lower body). The RE protocol in the present investigation had an average duration of 52 minutes and focused on the major muscle groups of the lower body. Furthermore, recent studies have shown that muscle glycogen stores can decrease by 20 to $40 \%$ during moderate intensity, high volume resistance exercise ranging from 36 to 50 total repetitions (Haff et al., 2000; MacDougall et al., 1999; Pascoe et al., 1993; Robergs et al., 1991; Tesch et al., 1996, 1998). In the current investigation, subjects performed an average of 89 to 136 total repetitions of the leg press exercise which is more than double the amount of repetitions of previous studies. Therefore, it is felt that the RE protocol used in the current investigation was sufficient to deplete muscle glycogen stores and demonstrate the effects of supplementation on performance. 
While the present investigation did not measure blood glucose or muscle glycogen levels throughout RE, results suggested a glycogen sparing effect occurred in the CHO-PRO and CHO groups which allowed significantly more repetitions and greater volume of work (non-significant) performed compared to the PLA group. However, several issues may explain why the CHO-PRO group did not significantly improve performance compared to the $\mathrm{CHO}$ only group. Since the phosphagen system is considered a primary source of energy during RE, fatigue may have occurred within a given set regardless of type of supplement consumed. Once phosphocreatine levels are depleted fatigue will occur during a given RE set in spite of maintained blood glucose and muscle glycogen levels. In addition, the accumulation of lactate and inorganic phosphates may have contributed to the onset of fatigue regardless of supplementation. Lactate accumulation interferes with muscle contraction by affecting calcium release from the sarcoplasmic reticulum, actin-myosin binding, and the production / breakdown of ATP (Housh et al., 2006). In addition, inorganic phosphate accumulation leads to muscular fatigue by affecting actin-myosin binding, myofibrillar calcium sensitivity for binding with troponin, calcium release from the sarcoplasmic reticulum, and calcium uptake by the sarcoplasmic reticulum (Westerblad et al., 2002).

Additionally, while protein can be converted to glucose via gluconeogenesis, the RE session may have not been long enough for gluconeogenesis to be a factor in energy production for the CHO-PRO group. Romano et al. (2006) stated that the additional protein from a CHO-PRO supplement was only a factor in energy production during long duration continuous aerobic endurance exercise. The discontinuous nature of the RE session (3 min rest periods), intake of carbohydrate after every other set, and potential 
slower absorption of protein may have not stimulated gluconeogenesis to help replenish blood glucose and muscle glycogen stores in the CHO-PRO group. Therefore, it is possible that the addition of protein may have not provided any additional benefits relative to energy production and resistance exercise performance for the CHO-PRO group when compared to the $\mathrm{CHO}$ group in the present investigation.

\subsection{PERCEIVED EXERTION}

Previous studies have shown that changes in blood glucose and muscle glycogen levels will alter perceived exertion during exercise (Noble and Robertson, 1996; Utter et al., 2007). It is felt that a reduction in blood glucose and muscle glycogen during exercise contributes to muscular fatigue, resulting in a greater perceived exertion (Utter et al., 2007). Ingesting a CHO supplement throughout exercise has been shown to maintain blood glucose and muscle glycogen levels leading to an attenuated perceived exertion response. Blomstrand et al. (1997) demonstrated that branched-chain amino acids (leucine, isoleucine, and valine) found in most protein supplements attenuated perceived exertion during exercise by influencing the synthesis, concentration, and release of neurotransmitters in the brain. This suggests that the use of CHO-PRO supplementation may attenuate perceived exertion during RE to a greater extent when compared to a traditional CHO only supplementation regimen.

In the present investigation RPE was measured after every exercise set in order to determine the effect of supplementation on feelings of discomfort or fatigue during the RE session. It was hypothesized that CHO-PRO supplementation would result in a 
comparatively greater attenuation of perceived exertion during RE compared to $\mathrm{CHO}$ supplementation or the PLA condition. Results revealed a significant difference in RPE$\mathrm{AM}$ at 25 and 50\% of total volume of work between the CHO-PRO and $\mathrm{CHO}$ groups. The CHO group had lower RPE-AM response at 25 and 50\% of total volume of work compared to the CHO-PRO group, and agrees with previous work suggesting carbohydrate availability can be a potential mediator of perceived exertion during exercise (Robertson et al., 1990; Utter et al., 1999 and Utter et al., 2007). In addition, Backhouse et al. (2005) demonstrated that CHO supplementation attenuated RPE response during aerobic endurance exercise. The lower RPE-AM response for the CHO group compared to the CHO-PRO group during the early stages of the exercise session may be due to a slightly lower absolute volume of work performed by these subjects. Previous research has shown that RPE is highly correlated with volume of work (Robertson et al., 2003). Therefore, the CHO group performed a lower absolute volume of work at 25 and $50 \%$ of the total volume, resulting in a corresponding lower RPE-AM response.

Also, the addition of protein may have slowed gastric emptying and the absorption of carbohydrate contributing to a higher RPE-AM response during the early stages of the exercise session. Generally, the digestion and absorption of protein is slow compared to carbohydrates, and the addition of protein to a carbohydrate meal slows the absorption of all nutrients (Brooks et al., 2004). However, limited research on effects of various nutritional supplements on gastric emptying and absorption rates has shown no significant difference between traditional $\mathrm{CHO}$ and CHO-PRO supplements (Seifert et al., 2009). 
No differences occurred in Session RPE between the CHO-PRO, CHO, and PLA groups. An interesting finding in the current investigation is that Session RPE for the PLA group was similar to the CHO-PRO and $\mathrm{CHO}$ groups despite performing significantly less repetitions and total volume of work during the resistance exercise session. Previous evidence suggests that carbohydrate availability acts as a mediator of perceived exertion during exercise (Robertson et al., 1990; Utter et al., 1999; Utter et al., 2007). While not directly measured in the present investigation, it is possible that the decreased levels of blood glucose and muscle glycogen in the PLA group contributed to a premature onset of fatigue compared to the CHO-PRO and $\mathrm{CHO}$ groups. An early onset of muscular fatigue potentially caused increased motor unit recruitment in an attempt to maintain power output. This in turn may have increased perceived exertion despite performing less work than the CHO-PRO and CHO groups. Although the PLA group performed less work than the CHO-PRO and CHO groups, perceived exertion may have been exacerbated due to these mechanisms surrounding muscular fatigue.

The results from this investigation suggest that the use of $\mathrm{CHO}-\mathrm{PRO}$ and $\mathrm{CHO}$ supplementation may attenuate perceived exertion which may allow an athlete to perform more work during a resistance exercise session. Over a given period of time, attenuated perceived exertion due to $\mathrm{CHO}-\mathrm{PRO}$ or $\mathrm{CHO}$ supplementation may help an athlete to improve muscular adaptations translating to improved power and force output and efficiency of exercises performed. Ultimately, this can contribute to improved sport specific skills and performance. 


\subsection{SALIVARY CORTISOL}

Moderate intensity, high volume RE increases cortisol levels (Crewther et al., 2008; McGuigan et al., 2004). While attempting to provide energy in response to declines in muscle glycogen and blood glucose, cortisol stimulates gluconeogenesis and peripheral release of substrates (Haff et al., 2003; Utter et al., 2007). Previous studies have shown that performing RE without supplementation will result in a significant post-exercise increase in salivary cortisol indicative of increased muscle protein breakdown (Crewther et al., 2008; McGuigan et al., 2004). A reduced post-exercise plasma cortisol response to RE following CHO-PRO supplementation has previously been shown (Baty et al., 2007; Bird et al., 2006; Williams et al., 2002). In addition, Utter et al. (1999) found that higher levels of plasma glucose due to $\mathrm{CHO}$ supplementation were associated with lower levels of cortisol during aerobic endurance exercise.

The present investigation hypothesized that CHO-PRO supplementation would result in a reduced post-exercise salivary cortisol response compared to $\mathrm{CHO}$ supplementation or PLA. On the contrary, the CHO-PRO group showed a 70\% increase in post-exercise salivary cortisol; however the differences were not statistically significant when compared to the $\mathrm{CHO}$ group. These findings are in contrast to previous

studies suggesting that the addition of protein to a carbohydrate supplement could suppress cortisol to a greater extent than carbohydrate alone (Saunders et al., 2004; Saunders et al., 2006; Ivy et al., 2003; Romano et al., 2006).

Similar to previous findings by Kraemer et al. (1998) that reported suppressed cortisol levels with CHO supplementation during resistance exercise, the post-exercise 
salivary cortisol response was significantly lower for the CHO group (20\% increase) compared to the PLA group (65\% increase). The response of the PLA group was similar to studies by Crewther et al. (2008) and McGuigan et al. (2004) where similar resistance training protocols resulted in a significant 47 to $97 \%$ increase in post-exercise salivary cortisol.

The increased salivary cortisol response of the CHO-PRO group may be due to a lower amount of carbohydrate per volume in the CHO-PRO supplement (15 g vs. $19 \mathrm{~g}$ of carbohydrate per 8 oz.). Since the CHO-PRO group is actually consuming less carbohydrate and performing a similar amount of work, the increased cortisol response may be an attempt to provide more energy via gluconeogenesis. In order to perform a similar volume of work as the $\mathrm{CHO}$ group, the CHO-PRO group may have needed to convert the recently ingested protein into glucose via gluconeogenesis in the liver.

It is also possible that the increased salivary cortisol response of the CHO-PRO group compared to the $\mathrm{CHO}$ group may have been due to variations in individual daily cortisol patterns. Cortisol levels follow a daily pattern that is affected by circadian rhythms, eating, and exercise, and are generally higher in the morning and decrease throughout the day (Brooks et al., 2004). Although all salivary cortisol measurements were taken in the afternoon between the hours of 1:00 and 4:00 pm in an attempt to minimize this issue, individual daily patterns still may have been a factor in the results. 


\section{$5.4 \quad$ LIMITATIONS}

The protocol used in this investigation contained a number of limitations that may have affected the results.

1. The sample size $(\mathrm{N}=27)$ of this study may be a significant limitation. The observed power for total volume of work and number of repetitions was 0.41 and 0.68 , respectively. A larger sample size $(\mathrm{N}=45+)$ is needed in order to further explain the effects of CHO-PRO and CHO supplementation on resistance exercise performance.

2. High variability in volume of work performed by the $\mathrm{CHO}$ group may have been a limitation in regards to finding significance. This variability was mainly due to a subject with a relatively high $1 \mathrm{RM}(477 \mathrm{~kg})$ who performed a high number of repetitions (156) and a subject with a relatively low 1 RM (123 kg) who performed a low number of repetitions (75).

3. The subject's individual effort during exercise testing may also be a significant limitation. Each subject performed a 1 RM strength test on the leg press machine in order to determine workload ( $70 \%$ of $1 \mathrm{RM}$ ) during the experimental protocol. If the subject did not provide a maximal effort during this initial test, the workload assigned during the experimental protocol may be insufficient, and could have affected RE performance. In addition, intra-individual variations in 
effort during the experimental protocol could have resulted in more or less repetitions performed regardless of the assigned nutritional supplement. Variations in intra-individual effort may have been due to factors such as lack of sleep, psychological stress, and low motivation.

4. Another potential limitation of this study was the subject's adherence to dietary guidelines. Subjects were encouraged to maintain their current dietary habits and avoid taking nutritional supplements of any kind two weeks prior to the experimental protocol. Subjects were also instructed to fast overnight prior to reporting to the laboratory in the morning for the standardized breakfast on the day of the experimental protocol. In addition, they were instructed not to eat any other foods between the standardized breakfasts and reported back to the lab for the early afternoon resistance exercise session. If subjects did not specifically follow any of these instructions, results of the study may have been compromised.

5. The supplementation protocol may have been a limitation for some subjects who participated in this investigation. The protocol required a pre-exercise supplement $\left(8 \mathrm{ml} \cdot \mathrm{kg}^{-1}\right)$ approximately 15 minutes prior to the RE session and between every other set (approximately every 7.5 minutes) during the exercise session ( $8 \mathrm{ml} \cdot \mathrm{kg}^{-1}$ divided into 8 supplements per hour). A few subjects mentioned they felt it was too much to drink in a short period of time, while most subjects had no complaints. 
6. While all the supplements were the same flavor (fruit punch), some subjects may have assumed they had a particular supplement based on the taste (or aftertaste) of the beverage. This may have affected subject performance during the RE session.

7. The lack of repeated measures experimental design may have also been a limitation in this investigation. A repeated measures design would require each subject undergo all three treatment conditions in a counterbalanced order. The use of a repeated measure design may have reduced the amount of variability between the treatment groups and increased the validity of the results.

8. The accuracy of the salivary cortisol measurements may have been a limitation in this investigation. Cortisol levels follow a daily pattern that is affected by circadian rhythms, eating and exercise. In general, cortisol levels are higher during the morning and decline later in the day, yet can increase in response to exercise and food intake (Brooks et al., 2004). Therefore, all cortisol measurements were taken in the afternoon between 1:00 and 4:00 pm in order to avoid the typically higher morning cortisol levels. Although the protocol was designed to minimize variations in the daily cortisol pattern, it is possible that individual variations in daily cortisol patterns may have affected the results of this investigation.

9. Additional cortisol samples may have been beneficial to examine the effect of supplementation on daily cortisol patterns. A morning cortisol sample could have been used to establish a baseline reference point. Additional post-exercise 
cortisol samples taken immediately post-exercise and 60 minutes post-exercise could have been used establish a more accurate cortisol pattern. These additional samples could have been used to better examine the influence of supplementation on the change in cortisol from baseline to post-exercise.

10. The collection of multiple salivary cortisol samples at each measurement time point could have helped determine the accuracy and reliability of the results. Although subjects were required to thoroughly rinse their mouth with water during the 30 minute post-exercise recovery prior to the post-exercise salivary cortisol measurement, it is possible that samples may have been contaminated due to leftover residue from the supplements.

\subsection{RECOMMENDATIONS FOR FUTURE RESEARCH}

Based on the limitations and results of this study, the following should be examined in future research regarding the use of $\mathrm{CHO}-\mathrm{PRO}$ and $\mathrm{CHO}$ supplementation during resistance exercise.

1. The results of this study leave a number of questions regarding the underlying physiological mechanisms such as: 1) the effect of RE on blood glucose and muscle glycogen levels; 2) the effect of CHO-PRO and CHO supplementation on maintaining blood glucose and muscle glycogen levels during RE and; 3) the effect of CHO-PRO and CHO supplementation on salivary cortisol levels during 
the entire RE session and post-exercise recovery period. Therefore, future research should focus on measuring blood glucose, muscle glycogen, and salivary cortisol levels before, during, and after consumption of CHO-PRO and CHO supplementation during resistance exercise. Measurement of these additional physiological variables may help to identify underlying mechanisms responsible for improvements in exercise performance.

2. The effects of CHO-PRO and $\mathrm{CHO}$ supplementation on recovery from resistance exercise should also be explored. Some evidence exists suggesting CHO-PRO supplementation may be more effective than $\mathrm{CHO}$ supplementation for improving muscular adaptations and reducing muscle soreness following a resistance exercise session (Baty et al., 2007). This would help researchers understand nutritional supplementation and its impact on recovery from resistance exercise and effects on sport performance.

3. Future research should consider the effects of CHO-PRO and $\mathrm{CHO}$ supplementation on affect and exercise enjoyment. This may provide additional insight into the potential psychological advantages of supplementation. If CHOPRO and CHO supplementation results in increased affect and exercise enjoyment then an athlete may have a more positive outlook regarding RE training sessions. A more enjoyable experience may result in improved compliance and more frequent RE sessions which can ultimately lead to improved muscular adaptations and exercise performance. 
4. Future research should consider the effects of different brands of CHO-PRO and CHO supplements found on the market today. Supplements are available in many forms and vary based on factors such as nutrient concentration (i.e. \% concentration of $\mathrm{CHO}$, PRO, vitamins, minerals, etc.) and delivery mechanism (i.e. gel, powder, bars, drinks, etc.). The differences in the formulation of a supplement may positively or negatively affect the supplements ability to provide benefits for a given exercise situation.

\subsection{CONCLUSIONS}

CHO-PRO and CHO supplements are popular methods of supplementation used by amateur and professional athletes. Research regarding these supplements effectiveness during resistance exercise has been inconsistent. This investigation demonstrated that both CHO-PRO and CHO supplementation before and during moderate intensity, high volume RE results in a significant increase in repetitions and volume compared to a PLA, suggesting the caloric content of both supplements contribute to maintaining blood glucose and sparing muscle glycogen for improved performance.

In addition, results of the present study suggest that CHO supplementation may be more effective than CHO-PRO supplementation for attenuating perceived exertion during the early stages of a moderate intensity, high volume RE session. This may lessen the intensity of effort and discomfort felt early in the RE session allowing training to continue for an extended period of time. In the long term, attenuated perceived exertion 
due to supplementation may help to improve resistance exercise performance and increase muscular adaptations leading to improved sport performance. Finally, this investigation demonstrated that $\mathrm{CHO}$ supplementation before and during resistance exercise may be more effective than CHO-PRO for reducing the post-exercise salivary cortisol response. The results of this investigation suggest that both CHO-PRO and CHO supplementation before and during RE can have a positive impact on exercise sessions.

Based on the results of this investigation, trainers and coaches should consider recommending the use of either a $\mathrm{CHO}$ or CHO-PRO supplement before and during moderate intensity, high volume RE sessions in order to improve performance. Future research should focus on the effects of $\mathrm{CHO}$ and CHO-PRO supplementation before, during, and after moderate intensity, high volume RE sessions to determine if either supplement is more advantageous for the recovery process. In addition, further exploration of the nature and mechanisms surrounding CHO-PRO and $\mathrm{CHO}$ supplementation may help to identify key muscular adaptations necessary for training and improved sport performance. 


\section{APPENDIX A}

\section{Orientation and anchoring procedures for OMNI-RES}

The investigator will read the following definition of RPE: “The perception of physical exertion is defined as the subjective intensity of effort, strain, discomfort, and/or fatigue that you feel during exercise”.

\section{The investigator will then read the following script to each subject prior to the RE} session: "We would like you to use these pictures to describe how your body feels during weightlifting exercise (show subject the OMNI-RES). You are going to perform resistance exercises using your upper and lower body. Please look at the person at the bottom of the scale who is performing a repetition using a light weight. If you feel like this person when you are lifting weights the exertion will be EXTREMELY EASY. In this case, you would respond with the number zero. Now look at the person at the top of the scale who is barely able to perform a repetition using a very heavy weight. If you feel like this person when you are lifting weights the exertion will be EXTREMELY HARD. In this case, you would respond with the number 10. If you feel somewhere in between Extremely Easy (0) and Extremely Hard (10), then give a number between 0 and 10. We will ask you to give a number that describes how your active muscles feel and then a number that describes how your whole body feels. Remember, there is no right or wrong numbers. Your number can change as you lift weights. Use both the pictures and the words to help select the numbers. Use any of the numbers to describe how you feel when lifting weights”. 
ID \#

\section{University of Pittsburgh Center for Exercise and Health-Fitness Research}

\section{Physical Activity Readiness Questionnaire (PAR-Q)}

Now I am going to ask you a few questions to determine if you are eligible to complete the resistance exercise session...

1. Has your doctor ever said that you have a heart condition and that you should only do physical activity recommended by a doctor?

No __ Yes _ _ If yes, specify:

2. Do you feel pain in your chest when you do physical activity?

No __ Yes _ _ If yes, specify:

3. In the past month, have you had chest pain when you were not doing physical activity?

No __ Yes __ If yes, specify:

4. Do you lose your balance because of dizziness or do you ever lose consciousness?

No __ Yes __ If yes, specify:

5. Do you have a bone or joint problem that could be made worse by a change in your physical activity?

$$
\text { No __ Yes _ _ If yes, specify: }
$$

6. Is your doctor currently prescribing drugs (for example, water pills) for a blood pressure or heart condition?

No __ Yes __ If yes, specify:

7. Do you know of any other reason why you should not do physical activity?

No __ Yes _ _ If yes, specify: 


\section{APPENDIX C}

ID \#

\section{University of Pittsburgh}

\section{Center for Exercise and Health-Fitness Research \\ MEDICAL HISTORY \\ YES}

1. History of heart problems, chest pain, or stroke?

2. Increased blood pressure?

3. Any chronic illness or condition?

4. Difficulty with physical exercise?

5. Advice from a physician not to exercise?

6. Recent surgery? (Last 12 months)

7. Pregnancy? (Now or within the last 3 months)

8. History of breathing or lung problems?

9. Muscle, joint, back disorder, or any previous injury still affecting you?

10. Diabetes or thyroid conditions?

11. Cigarette smoking habit?

12. Increased blood cholesterol?

13. History of heart problems in your immediate family?

14. Hernia or any condition that may be aggravated by lifting weights?

15 . Do you have any condition limiting your movement?

16. Are you aware of being allergic to any drugs or insect bites?

17. Do you have asthma?

18. Do you have epilepsy, convulsions, or seizures of any kind?

19. Do you follow any specific diet?

Please explain in detail any "YES” answers:

Family History

Has any member of you family had any of those listed above?
NO

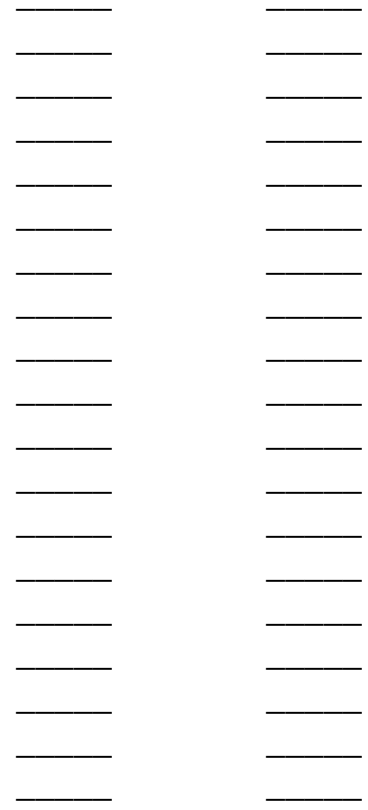




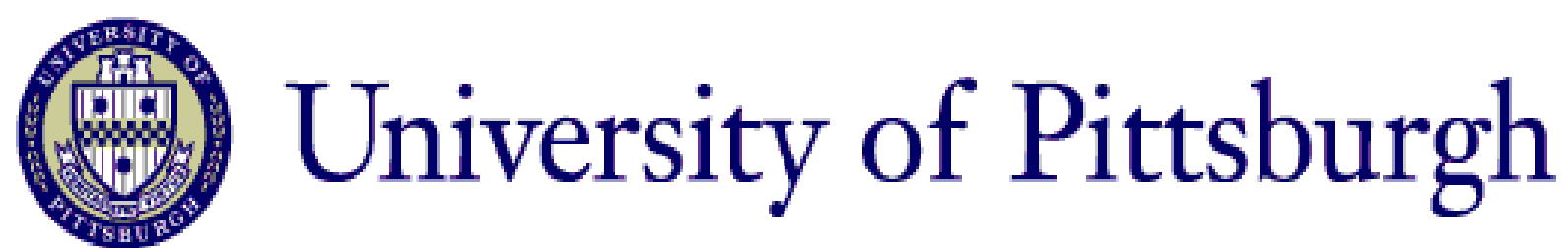

RESEARCH STUDY

\author{
DEPARTMENT OF HEALTH AND PHYSICAL ACTIVITY \\ CENTER FOR EXERCISE AND HEALTH-FITNESS RESEARCH
}

University of Pittsburgh Department of Health and Physical Activity is now recruiting healthy, recreationally active males (18-30 years old) for a research study to determine the effects of nutritional supplementation on resistance exercise performance, perceived exertion, and salivary cortisol

\title{
Each participant will complete:
}

Two exercise sessions using a seated leg press machine. The first session involves the determination of maximal strength. The second session involves performing multiple sets of the leg press exercise to fatigue.

\section{To be eligible you must:}

1. Be healthy

2. Participate in weight training at least 2 but not more than 4 times per week over the previous 6 months.

3. Not knowingly taking any performance enhancing substances

4. Not participating in collegiate or professional athletics

Upon completion, subjects will receive $\$ 20.00$

For more information and to see if you qualify, call Mike Welikonich at 412-648-8251 or e-mail miw11@pitt.edu for more details. 


\section{APPENDIX E}

\section{University of Pittsburgh}

School of Education

Health, Physical and Recreation Education

Center for Exercise and Health-Fitness Research

\section{INFORMED CONSENT}

\section{TITLE: EFFECT OF CARBOHYDRATE-PROTEIN SUPPLEMENTATION ON RESISTANCE EXERCISE PERFORMANCE, PERCEIVED EXERTION, AND SALIVARY CORTISOL}

PRINCIPAL INVESTIGATOR:

\section{CO-INVESTIGATORS:}

Michael J. Welikonich, M.S.

Graduate Student Assistant, Center for

Exercise and Health-Fitness Research

140 Trees Hall

Pittsburgh, PA 15216

Phone: (412) 648-8251

Email: miw11@pitt.edu

Department of Health and Physical Activity

School of Education

Elizabeth F. Nagle, Ph.D.

Assistant Professor and Assistant Director,

Center for Exercise and Health Fitness

Research

149 Trees Hall

Phone: (412) 648-8265 Fax: (412) 648-7092

Email: nagle@pitt.edu

Department of Health and Physical Activity

School of Education

Robert J. Robertson, Ph.D.

Professor and Co-Director, Center for

Exercise and Health-Fitness Research

107 Trees Hall

Pittsburgh, PA 15216

Phone: (412) 648-8251 Fax: (412) 648-7092

Email: rrobert@pitt.edu

Department of Health and Physical Activity

School of Education

SOURCE OF SUPPORT: School of Education Research Grant 


\section{Why is this research being done?}

Nutritional supplementation is critical to many athletes, trainers, and coaches who seek safe and legal methods to improve exercise performance. Carbohydrate-protein and carbohydrate beverages are two popular nutritional supplements currently used by many athletes. Evidence suggests that consumption of a carbohydrate-protein supplement compared to a carbohydrate supplement may improve performance and reduce feelings of exertion during aerobic exercise. By reducing feelings of fatigue or exertion during an exercise session an athlete will be able to perform more work which can result in improved muscular adaptations. Yet, it remains unclear whether carbohydrate-protein supplementation can improve performance or decrease feeling of fatigue during resistance exercise. Therefore, the purpose of this investigation is to determine the effect of carbohydrate-protein versus carbohydrate supplementation and placebo on performance and feelings of exertion during resistance exercise.

\section{Who is being asked to take part in this research study?}

Forty five college aged (18 to 30 yrs) healthy males who participate in recreational strength training will be recruited from the university community to participate in this investigation. You have been invited to participate in the research study because an initial interview at the time of recruitment revealed that you are healthy, have normal body weight, are not knowingly taking any performance enhancing substances, have not used nutritional supplements of any kind for at least the last three weeks, and performed resistance exercise at least twice but not greater than four times per week over the previous 6 months. If you have a cardiovascular (heart), musculoskeletal (muscle or bone), and/or metabolic disease (i.e. diabetes), currently being treated for psychological disorder and/or knowingly taking any performance enhancing substances including anabolic steroids you will not be eligible to participate in this research study.

\section{What procedures will be performed for research purposes?}

You will randomly receive a carbohydrate-protein, carbohydrate, or placebo beverage 15 to 30 minutes before a resistance exercise session, between every other set during the exercise session, and immediately post exercise. You will complete a resistance exercise session consisting of multiple sets of seated leg press exercise starting with 1 set of 10 repetitions at 40,50, and 60\% of your 1 repetition maximum, continuing with multiple sets of 8 to 10 repetitions at $70 \%$ of your 1 repetition maximum until fatigue is reached (i.e. unable to complete 8 repetitions). Three minute rest intervals will occur between all sets. You will be asked to rate feelings of exertion for the active muscle group (legs) and overall body immediately following each set of the seated leg press exercise. Additionally, you will rate an overall feeling of exertion for the entire exercise session 30 minutes after the resistance exercise session is completed. Salivary cortisol samples will be collected approximately 15 minutes prior to exercise and 30 minutes post-exercise.

University Of Pittsburgh Institutional Review Board
Approval Date: 4/28/2009

Renewal Date: 4/6/2010
Participants Initials

IRB \#: PRO09030005 
If an abnormal response occurs during exercise, such as severe muscle or joint pain, the test will be immediately stopped and you will be given proper medical attention. Emergency equipment will be on site for all testing procedures and staff personnel are certified in CPR and First Aid by the American Red Cross. All procedures will take place in the Human Energy Research Laboratory at the Center for Exercise and HealthFitness Research located in Trees Hall at the University of Pittsburgh. All testing sessions will be administered by the primary investigator and trained investigators from the Center for Exercise and Health-Fitness Research.

\section{Day 1: Initial Assessment Procedures:}

1. Before starting the study protocol, you will have up to 10 minutes to read and complete this informed consent document. In addition, you will complete a medical history form and a physical activity readiness questionnaire (PAR-Q). Both forms will take less than five minutes to complete.

2. You will be assessed for height, weight, and body composition. Body height $(\mathrm{cm})$ and weight (kg) will be measured using a standard physician scale. Body composition will be determined using a Tanita body fat analyzer. This is a noninvasive pain-free procedure for assessing body fat and muscle that transmits a lowgrade electrical impulse through the body. Your height, age, and gender will be entered into the Tanita analyzer and the "standard" mode for calculating body fat will be used. You will be instructed to remove your shoes and socks and to stand on the Tanita sensors with your bare feet until a body fat reading is determined.

3. Based on the information you provide on the medical history and physical activity questionnaire, if you do not have any conditions that would limit your ability to exercise, you will complete the first testing session in order to determine your 1 repetition maximum for the leg press exercise. You will perform an initial warm-up set of 10 repetitions at a self selected light weight on a leg press machine. Following a 1 minute rest period, weight will be increased by $10-20 \%$ for the leg press exercise where 3 to 5 repetitions will be performed. Following a 2 minute rest period, a near-maximum load will be estimated by the trained investigator and you will perform up to 3 repetitions. Following a 3 minute rest period, the load will again be increased by $10-20 \%$. You will then attempt a 1 repetition maximum. If successful, you will rest for 3 minutes and attempt a weight increased by the same methods as previously stated. Once you are unable to lift a weight, the highest weight lifted successfully will be your 1 repetition maximum. Ideally, this will be measured within five testing sets in order to prevent performance decrements due to fatigue.

University Of Pittsburgh Institutional Review Board
Approval Date: 4/28/2009

Renewal Date: 4/6/2010
Participants Initials

IRB \#: PRO09030005 
4. Prior to exercise testing you will undergo an orientation session to ensure you are able to use the OMNI Resistance Exercise Scale to rate your feeling of exertion. The investigator will read to you the following definition of ratings of perceived exertion: "Rating of perceived exertion is defined as a measure of the subjective intensity of effort, strain, discomfort, and/or fatigue that you feel during exercise.” Other scaling procedures for the OMNI scale will be administered to you prior to the 1 repetition maximum test.

5. For the 7 days prior to the exercise trial on day 2, you will be encouraged to maintain your current dietary habits and avoid taking nutritional supplements of any kind. You will be instructed to record your food intake for the 3 days prior to the exercise trial in a food journal provided to you by the investigator. Your three day diet records will be analyzed using a computerized dietary assessment program to determine any major nutritional differences between subjects.

\section{Day 2: Supplementation Protocol}

1. Approximately 7 days following the initial assessment, you will report to the Human Energy Research Lab between the hours of 8:00 and 10:00 am having fasted overnight for 12 hours. You will be provided a standardized liquid meal which will serve as a control for any dietary differences that may exist among the subjects. The standardized liquid meal will contain: 1) 50\% of energy as carbohydrate; 2) 34\% as fat; and 3)16\% as protein.

2. You will then report back to the lab between 1:00 and 3:00 pm not having ingested energy in any form for 5 hours. You will also be instructed not to chew gum or brush your teeth for 30 minutes before reporting back to the lab.

3. You will be seated for 15 minutes prior to the collection of the resting salivary cortisol sample. For each salivary cortisol measurement, you will deposit saliva into a sterile container with the aid of a straw until the container is half full. All samples will be labeled and refrigerated until sent to the testing laboratory to be analyzed.

4. After the salivary cortisol sample is collected, you will receive either a carbohydrate protein supplement, carbohydrate supplement or placebo 15 minutes prior to the start of the exercise session and immediately post-exercise. You will also receive supplementation between every other set (approximately every $7.5 \mathrm{~min}$ ) throughout the exercise session. All three supplements will be similar in appearance and taste. The amount of supplement you receive will be based on your body weight.

University Of Pittsburgh Institutional Review Board
Approval Date: 4/28/2009

Renewal Date: 4/6/2010
Participants Initials

IRB \#: PRO09030005 


\section{Day 2: Resistance Exercise Protocol}

1. The exercise protocol will consist of performing multiple sets of seated leg press exercise until fatigue is reached. Fatigue will be considered the point at which you cannot complete the required $8-10$ repetitions or can no longer continue due to exhaustion.

2. You will begin with a warm-up consisting of 5 minutes of riding a Monark Cycle Ergometer with low resistance $(0.5 \mathrm{~kg})$ at $50 \mathrm{rpm}$ followed by standardized lower body static stretching supervised by a trained investigator. A warm-up set will follow where you will perform one set of 10 repetitions at $40 \%$ of your 1 repetition maximum as determined by a trained investigator. For the subsequent 2 warm-up sets, you will perform 1 set of 10 repetitions at both 50 and $60 \%$ of 1 repetition maximum.

3. Following the warm-up, you will perform multiple sets of 8 to 10 repetitions at $70 \%$ of your 1 repetition maximum until fatigue. A 3 minute rest period, timed by a trained investigator, will follow each set. Supplementation will occur during this rest period after every other set. Ratings of perceived exertion for the active muscle and overall body will be obtained immediately after each set of seated leg press exercise using the OMNI Resistance Exercise Scale. Blood pressure will be measured between every other set and heart rate will be monitored continually during the exercise protocol. A rating of perceived exertion for the entire exercise session and salivary cortisol measurement will be obtained with you in a seated position 30 minutes following the resistance exercise session.

\section{What are the possible risks, side effects, and discomforts of this research study?}

As a result of these tests and training you may experience local muscle fatigue or soreness which should dissipate within 24 to 48 hours. In rare instances there may be an abnormal heart rate response (failure to increase with increased exercise intensity), blood pressure response (systolic $>260 \mathrm{mmHg}$ or diastolic $>115 \mathrm{mmHg}$ ), fainting, and heart attack (less than 1 out of 100 people or 1\%). If an abnormal response occurs during exercise, the test will be immediately terminated and the subject given proper medical attention. There is also a chance of a musculoskeletal injury due to attempting maximum lifts.

\section{What are possible benefits from taking part in this study?}

You will likely receive little direct benefit from taking part in this research study. However, you will receive information regarding your maximal strength, percent body fat, and the importance of nutritional supplementation on exercise performance.

University Of Pittsburgh Institutional Review Board
Approval Date: 4/28/2009

Renewal Date: 4/6/2010
Participants Initials

IRB \#: PRO09030005 
If I agree to take part in this research study, will I be told of any new risks that may be found during the course of the study?

You will be promptly notified if, during the conduct of this research study, any new information develops which may cause you to change your mind about continuing to participate.

Will my insurance provider or I be charged for the costs of any procedures performed as part of this research study?

Neither you, nor your insurance provider, will be charged for the costs of any procedures performed for the purpose of this research study.

Will I be paid if I take part in this research study?

You will be paid $\$ 20$ upon completion of the two trials. There will be no partial compensation for completion of only the first trial.

\section{Who will pay if I am injured as a result of taking part in this study?}

University of Pittsburgh researchers and their associates who provide services at UPMC recognize the importance of your voluntary participation in their research studies. These individuals and their staffs will make reasonable efforts to minimize, control, and treat any injuries that may arise as a result of this research. If you believe that you are injured as a result of the research procedures being performed, please contact immediately the Principal Investigator or one of the Co-Investigators listed on the first page of this form.

Emergency medical treatment for injuries solely and directly related to your participation in this research study will be provided to you by the hospitals of the UMPC.

It is possible that the UPMC may bill your insurance provider for the costs of this emergency treatment, but none of these costs will be charged directly to you. If your research-related injury requires medical care beyond this emergency treatment, you will be responsible for the cost of this follow-up unless otherwise specifically stated below. There is no plan for monetary compensation. You do not, however, waive any legal rights by signing this form.

University Of Pittsburgh Institutional Review Board
Approval Date: 4/28/2009

Renewal Date: 4/6/2010
Participants Initials

IRB \#: PRO09030005 
Who will know about my participation in this research study?

Any information about you obtained from this research will be kept as confidential (private) as possible. All records related to your involvement in this research study will be stored in a locked file cabinet. Your identity on these records will be indicated by a case number rather than by your name, and the information linking these case numbers with your identity will be kept separate from the research records. You will not be identified by name in any publication of the research results unless you sign a separate consent form giving your permission (release).

Will this research study involve the use or disclosure of my identifiable medical information?

This research study will not involve the use or disclosure of any identifiable medical information.

Who will have access to identifiable information related to my participation in this research study?

In addition to the investigators listed on the first page of this authorization (consent) form and their research staff, the following individuals will or may have access to identifiable information related to your participation in this research study:

- Authorized representatives of the University of Pittsburgh Research Conduct and Compliance Office may review your identifiable research information for the purpose of monitoring the appropriate conduct of this research study.

- In unusual cases, the investigators may be required to release identifiable information related to your participation in this research study in response to an order from a court of law. If the investigators learn that you or someone with whom you are involved is in serious danger or potential harm, they will need to inform, as required by Pennsylvania law, the appropriate agencies.

- Authorized people sponsoring this research study, because they need to make sure that the information collected is correct, accurate, and complete, and to determine the results of this research study.

For how long will the investigators be permitted to use and disclose identifiable information related to my participation in this research study?

The investigators may continue to use and disclose, for the purposes described above, identifiable information related to your participation in this research study for a minimum of six years after final reporting or publication of a project.

University Of Pittsburgh Institutional Review Board
Approval Date: 4/28/2009

Renewal Date: 4/6/2010
Participants Initials

IRB \#: PRO09030005 


\section{Is my participation in this research study voluntary?}

Your participation in this research study, to include the use and disclosure of your identifiable information for the purposes described above, is completely voluntary. (Note, however, that if you do not provide your consent for the use and disclosure of your identifiable information for the purposes described above, you will not be allowed, in general, to participate in this research study.) Whether or not you provide your consent for participation in this research study will have no affect on your current or future relationship with the University of Pittsburgh. Whether or not you provide your consent for participation in this research study will have no effect on your current or future medical care at a UPMC hospital or affiliated health care provider or your current or future relationship with a health care insurance provider. If you are a student, the decision to participate or not participate in this study will have no influence on class standing or grades.

\section{May I withdraw, at a future date, my consent for participation in this research study?}

You may withdraw, at any time, your consent for participation in this research study, to include the use and disclosure of your identifiable information for the purposes described above. Any identifiable research information recorded for, or resulting from, your participation in this research study prior to the date that you formally withdrew your consent may continue to be used and disclosed by the investigators for the purposes described above.

To formally withdraw your consent for participation in this research study you should provide a written and dated notice of this decision to the principal investigator of this research study at the address listed on the first page of this form.

Your decision to withdraw your consent for participation in this research study will have no effect on your current of future relationship with the University of Pittsburgh. Your decision to withdraw your consent for participation in this research study will have no effect on your current of future medical care at a UPMC hospital or affiliated health care provider or your current or your future relationship with a health care insurance provider.

\section{If I agree to take part in this research study, can I be removed from the study without my consent?}

It is possible that you may be removed from the research study by the researchers to protect your safety or if you are unable or unwilling to complete the research protocol.

University Of Pittsburgh Institutional Review Board
Approval Date: 4/28/2009

Renewal Date: 4/6/2010
Participants Initials

IRB \#: PRO09030005 


\section{VOLUNTARY CONSENT}

All of the above has been explained to me and all of my questions have been answered. I understand that any future questions I have about this research study during the course of this study, and that such future questions will be answered by the investigators listed on the first page of this consent document at the telephone numbers given. Any questions I have about my rights as a research subject will be answered by the Human Subject Protection Advocate of the IRB Office, University of Pittsburgh (1-866212-2668). By signing this form, I agree to participate in this research study.

$\overline{\text { Participant's Name (Print) }}$

Participant’s Signature

Date

\section{CERTIFICATION OF INFORMED CONSENT}

I certify that I have explained the nature and purpose of this research study to the above-named individual, and I have discussed the potential benefits, and possible risks associated with participation. Any questions the individual has about this study have been answered, and we will always be available to address future questions as they arise. I further certify that no research component of this protocol was begun until after this consent form was signed.

Printed Name of Person Obtaining Consent

Role in Research Study

Signature of Person Obtaining Consent

Date

University Of Pittsburgh Institutional Review Board
Approval Date: 4/28/2009

Renewal Date: 4/6/2010
Participants Initials

IRB \#: PRO09030005 


\section{BIBLIOGRAPHY}

Backhouse, S. H., Bishop, N. C., Biddle, S. J., \& Williams, C. (2005). Effect of Carbohydrate and Prolonged Exercise on Affect and Perceived Exertion. Medicine and Science in Sports and Exercise , 37 (10), 1768-1773.

Baechle, T. R., \& Earle, R. W. (2000). Essentials of Strength Training and Conditioning. Champaign, IL: Human Kinetics.

Baty, J. J., Hwang, H., Ding, Z., Bernard, J. R., Wang, B., Kwon, B., et al. (2007). Effect of a carbohydrate and protein supplement on resistance exercise performance, hormonal response, and muscle damage. Journal of Strength and Conditioning Research , 21 (2), 321-329.

Beaven, C. M., Gill, N. D., \& Cook, C. J. (2008). Salivary testosterone and cortisol responses in professional rugby players after four resistance exercise protocols. Journal of Strength and Conditioning Research , 22 (2), 426-432.

Biolo, G., Maggi, S. P., Williams, B. D., Tipton, K. D., \& Wolfe, R. R. (1995). Increased rates of muscle protein turnover and amino acid transport after resistance exercise in humans. American Journal of Physiology , 268, E514-E520.

Biolo, G., Tipton, K. D., Klein, S., \& Wolfe, R. R. (1997). An abundant supply of amino acids enhances the metabolic effect of exercise on muscle protein. American Journal of Physiology, 36, E122-E129.

Bird, S. P., Tarpenning, K. M., \& Marino, F. E. (2006). Effects of liquid carbohydrate/essential amino acid ingestion on acute hormonal response during a single bout of resistance exercise in untrained men. Nutrition , 22, 367-375.

Bird, S. P., Tarpenning, K. M., \& Marino, F. E. (2006). Independent and combined effects of liquid carbohydrate/essential amino acid ingestion on hormonal and muscular adaptations following resistance training in untrained men. European Journal of Applied Physiology, 97, 225-238.

Bird, S. P., Tarpenning, K. M., \& Marino, F. E. (2006). Liquid carbohydrate/essential amino acid ingestion during short term bout of resistance exercise suppresses myofibrillar protein degradation. Metabolism Clinical and Experimental , 55, 570-577. 
Blomstrand, E. (2001). Amino acids and central fatigue. Amino Acids , 20, 25-34.

Blomstrand, E., Hassmen, P., Ek, S., Ekblom, B., \& Newsholme, E. A. (1997). Influence of ingesting a solution of branched-chain amino acids on perceived exertion during exercise. Acta Physiol Scand , 159, 41-49.

Borg, G. A. (1982). Psychophysical basis of percieved exertion. Medicine and Science in Sports and Exercise , 14 (5), 377-381.

Brooks, G. A., Fahey, T. D., White, T. P., \& Baldwin, K. M. (2004). Exercise Physiology: Human bioenergetics and its applications. New York, NY: McGraw-Hill.

Crewther, B., Cronin, J., Keogh, J., \& Cook, C. (2008). The salivary testosterone and cortisol response to three loading schemes. Journal of Strength and Conditioning Research , 22 (1), 250-255.

Day, M. L., McGuigan, M. R., Brice, G., \& Foster, C. (2004). Monitoring exercise intensity during resistance exercise training using the session RPE scale. Journal of Strength and Conditioning Research , 18 (2), 353-358.

Egan, A. D., Winchester, J. B., Foster, C., \& McGuigan, M. R. (2006). Using session RPE to monitor different methods of resistance exercise. Journal of Sports Science and Medicine (5), 289-295.

Fleck, S. J., \& Kraemer, W. J. (2004). Designing Resistance Training Programs. Champaign, IL: Human Kinetics.

Foster, C., Florhaug, J. A., Franklin, J., Gottschall, L., Hrovatin, L. A., Parker, S., et al. (2001). A new approach to monitoring exercise training. Journal of Strength and Conditioning Research , 15 (1), 109-115.

Foster, C., Florhaug, J. A., Franklin, J., Gottschall, L., Hrovatin, L. A., Parker, S., et al. (2001). A New Approach to Monitoring Exercise Training. Journal of Strength and Conditioning Research , 15 (1), 109-115.

Gallagher, P., Leitch, M. M., Massey, A., McAllister-Williams, \& Young, A. H. (2006). Assessing cortisol and dehydroepiandrosterone (DHEA) in saliva: effects of collection method. Journal of Psychopharmacology , 20 (5), 643-649.

Gearhart, R. F., Goss, F. L., Lagally, K. M., Jakicic, J. M., Gallagher, J., Gallagher, K. I., et al. (2002). Ratings of Perceived Exertion in Active Muscle During High-Intensity and Low-Intensity Resistance Exercise. Journal of Strength and Conditioning Research , 16 (1), 87-91. 
Gorzansky, W. S., Lynn, J. S., Laudenslager, M. L., \& Kohrt, W. M. (2005). Salivary cortisol determined by enzyme immunoassay is preferable to serum total cortisol for assessment of dynamic hypothalamic-pituitary-adrenal axis activity. Clinical Endocrinology , 63, 336-341.

Haff, G. G., Koch, A. J., Potteiger, J. A., Kuphal, K. E., Magee, L. M., Green, S. B., et al. (2000). Carbohydrate supplementation attenuates muscle glycogen loss during acute bouts of resistance exercise. Journal of Sport Nutrition and Exercise Metabolism , 10 (3), 326-339.

Haff, G. G., Lehmkuhl, M. J., McCoy, L. B., \& Stone, M. H. (2003). Carbohydrate Supplementation and Resistance Training. Journal of Strength and Conditioning Research , 17 (1), 187-196.

Hepburn, D., \& Maughan, R. J. (1982). Glycogen availability as a limiting factor in performance and isometric exercise. Journal of Physiology , 342, 52-53.

Hollander, D. B., Durand, R. J., Trynicki, J. L., Larock, D., Castracane, D., Hebert, E. P., et al. (2003). RPE, Pain, and Physiological Adjustment to Concentric and Eccentric Contractions. Medicine and Science in Sports and Exercise , 35 (6), 1017-1025.

Housh, T. J., Housh, D. J., \& Devries, H. A. (2006). Applied Exercise \& Sport Physiology. Scottsdale, AZ: Holcomb Hathaway.

Ivy, J. L., Res, P. T., Sprague, R. C., \& Widzer, M. O. (2003). Effect of a carbohydrateprotein supplement on endurance performance during exercise of varying intensity. Journal of Sport Nutrition and Exercise Metabolism , 13, 382-395.

Jacobs, I., Kaiser, P., \& Tesch, P. (1981). Muscle strength and fatigue after selective glycogen depletion in human skeletal muscle fibers. European Journal of Applied Physiology , 46 (1), 47-53.

Kulik, J. R., Touchberry, C. D., Kawamore, N., Blumert, P. A., Crum, A. J., \& Haff, G. G. (2008). Supplemental carbohydrate ingestion does not improve performance of highintensity resistance exercise. Journal of Strength and Conditioning Research , 00, 1-7.

Lagally, K. M., \& Robertson, R. J. (2006). Construct validity of the OMNI resistance exercise scale. Journal of Strength and Conditioning Research , 20 (2), 252-256.

Lagally, K. M., McCaw, S. T., Young, G. T., Medema, H. C., \& Thomas, D. Q. (2004). Ratings of perceived exertion and muscle activity during the bench press exercise in recreational and novice lifters. Journal of Strength and Conditioning Research , 18 (2), 359-364. 
Lagally, K. M., Robertson, R. J., Gallagher, K. I., Goss, F. L., Jakicic, J. M., Lephart, S. M., et al. (2002). Perceived exertion, electromyography, and blood lactate during acute bouts of resistance exercise. Medicine and Science in Sports and Exercise , 34 (3), 552559.

MacDougall, J. D., Ray, S., Sale, D. G., McCartney, N., Lee, P., \& Garner, S. (1999). Muscle substrate utilization and lactate production during weightlifting. Canadian Journal of Applied Physiology , 24 (3), 209-215.

McArdle, W. D., Katch, F. I., \& Katch, V. L. (2001). Exercise Physiology: Energy, Nutrition, and Human Performance. Baltimore, MD: Lippincott Williams \& Williams.

McGuigan, M. R., Egan, A. D., \& Foster, C. (2004). Salivary cortisol responses and perceived exertion during high intensity and low intensity bouts of resistance exercise. Journal of Sports Science and Medicine , 3, 8-15.

Neufer, P. D., Costill, D. L., Flynn, M. G., Kirwan, J. P., Mitchell, J. B., \& Houmard, J. (1987). Improvements in exercise performance: Effects of carbohydrate feedings and diet. Journal of Applied Physiology , 62 , 983-988.

Noble, B. J., \& Robertson, R. J. (1996). Perceived Exertion. Champaign, IL: Human Kinetics.

Pascoe, D. D., Costill, D. L., Fink, W. J., Robergs, R. A., \& Zachwieja, J. J. (1993). Glycogen resynthesis in skeletal muscle following resistance exercise. Medicine and Science in Sports and Exercise , 25 (3), 349-354.

Peters, J. R., Walker, F., Riad-Fahmy, D., \& Hall, R. (1982). Salivary cortisol assays for assessing pituitary-adrenal reserve. Clinical Endocrinology , 17, 583-592.

Phillips, S. M., Tipton, K. D., Aarsland, A., Wolf, S. E., \& Wolfe, R. R. (1997). Mixed muscle protein synthesis and breakdown after resistance exercise in humans. American Journal of Physiology, 273, E99-E107.

Phillips, S. M., Tipton, K. D., Ferrando, A. A., \& Wolfe, R. R. (1999). Resistance training reduces the acute exercise-induced increase in muscle protein turnover. American Journal of Physiology , 273, E118-E124.

Ramano-Ely, B. C., Todd, M. K., Saunders, M. J., \& Laurent, T. S. (2006). Effect of an isocaloric carbohydrate-protein antioxidant drink on cycling perforamcne. Medicine and Science in Sports and Exercise , 38 (9), 1608-1616.

Rasmussen, B. B., Tipton, K. D., Miller, S. L., Wolf, S. E., \& Wolfe, R. R. (2000). An oral essential amino acid-carbohydrate supplement enhances muscle protein anabolism after resistance exercise. Journal of Applied Physiology , 88, 386-392. 
Robergs, R. A., Pearson, D. R., Costill, D. L., Fink, W. J., Pascoe, D. D., Benedict, M. A., et al. (1991). Muscle glycogenolysis during differing intensities of weight-resistance exercise. Journal of Applied Physiology, 70 (4), 1700-1706.

Robertson, R. J. (2004). Perceived Exertion for Practitioners. Champaign, IL: Human Kinetics.

Robertson, R. J., \& Noble, B. J. (1997). Perception of physical exertion: Methods, mediators, and applications. Exercise and Sports Science Reviews , 25, 407-452.

Robertson, R. j., Goss, F. L., Rutkowski, J., Lenz, B., Dixon, C., Timmer, J., et al. (2003). Concurrent Validation of the OMNI Perceived Exertion Scale for Resistance Exercise. Medicine and Science in Sports and Exercise , 35 (2), 333-341.

Robertson, R. J., Nixon, P. A., Caspersen, C. J., Metz, K. F., Abbott, A., \& Goss, F. L. (1992). Abatement of exertional perceptions following dynamic exercise: physiological mediators. Medicine and Science in Sports and Exercise , 24 (3), 346-353.

Robertson, R. J., Stanko, R. T., Goss, F. L., Spina, R. J., Reilly, J. J., \& Greenawalt, K. D. (1990). Blood glucose extraction as a mediator of perceived exertion during prolonged exercise. European Journal of Applied Physiology , 61, 100-105.

Roy, B. D., Tarnopolsky, M. A., MacDougall, J. D., Fowles, J., \& Yarasheski, K. E. (1997). Effect of glucose supplement timing on protein metabolism after resistance training. Journal of Applied Physiology , 82, 1882-1888.

Saunders, M. J., Kane, M. D., \& Todd, M. K. (2004). Effects of carbohydrate-protein beverage on cycling endurance and muscle damage. Medicine and Science in Sports and Exercise , 36 (7), 1233-1238.

Seifert, J. G., Schapman, J., Little, T. L., Robins, A., Thompson, D. G., \& Portman, R. (2009). Gastric Emptying Characteristics of Carbohydrate/Protein and Carbohydrate Only Sports Drinks during Moderate Intensity Exercise. Medicine and Science in Sports and Exercise , 41 (5).

Suminski, R. R., Robertson, R. J., Arslanian, S., Kang, J., Utter, A. C., DaSilva, S. G., et al. (1997). Perception of effort during resistance exercise. Journal of Strength and Conditioning Research , 11 (4), 261-265.

Sweet, T. W., Foster, C., McGuigan, M. R., \& Brice, G. (2004). Quantitation of resistance training using the session rating of perceived exertion method. Journal of Strength and Conditioning Research , 18 (4), 796-802. 
Tesch, P. A., Ploutz-Snyder, L. L., Ystrom, L., Castro, M., \& Dudley, G. (1998). Skeletal muscle glycogen loss evoked by resistance exercise. Journal of Strength and Conditioning Research , 12 (2), 67-73.

Tipton, K. D., \& Wolfe, R. R. (1998). Exercise-induced changes in protein metabolism. Acta Physiologica Scandinavica , 162, 377-387.

Tipton, K. D., Rasmussen, B. B., Miller, S. L., Wolf, S. E., Owens-Stovall, S. K., Petrini, B. E., et al. (2001). Timing of amino acid-carbohydrate ingestion alters anabolic response of muscle to resistance exercise. American Journal of Physiology , 281, E197-E206.

Tsintzas, K., \& Williams, C. (1998). Human muscle glycogen metabolism during exercise. Sports Medicine , 25 (1), 7-23.

Umeda, T., Hiramatsu, R., Iwaoka, T., Shimada, T., Miura, F., \& Sato, T. (1981). Use of saliva for monitoring unbound free cortisol levels in serum. Clinica Chimica Acta , 110, 245-253.

Utter, A. C., Kang, J., Neiman, D. C., Brown, V. A., Dumke, C. L., McAnulty, S. R., et al. (2005). Carbohydrate supplementation and perceived exertion during resistance exercise. Journal of Strength and Conditioning Research , 19 (4), 939-943.

Utter, A. C., Kang, J., Nieman, D. C., Dumke, C. L., McAnulty, S. R., \& McAnulty, L. S. (2007). Carbohydrate attenuates perceived exertion during intermittent exercise and recovery. Medicine and Science in Sports and Exercise , 39 (5), 880-885.

Utter, A. C., Kang, J., Nieman, D. C., Williams, F., Robertson, R. J., Henson, D. A., et al. (1999). Effect of carbohydrate ingestion and hormonal responses on ratings of perceived exertion during prolonged cycling and running. European Journal of Applied Physiology , 80, 92-99.

Van Loon, L. J., Saris, W. H., Kruijshoop, M., \& Wagenmakers, A. J. (2000). Maximizing post-exercise muscle glycogen synthesis: carbohydrate supplementation and the application of amino acid and protein hydrolysate mixtures. American Journal of Clinical Nutrition , 72 (1), 106-111.

Volek, J. S. (2004). Inflluence of Nutrition on Responses to Resistance Training. Medicine and Science in Sports and Exercsie , 36 (4), 689-696.

Westerblad, H., Allen, D. G., \& Lannergren, J. (2002). Muscle fatigue: Lactic acid or inorganic phosphate the major cause. News Physiol Sci , 17, 17-21.

Williams, A. G., Ismail, A. N., Sharma, A., \& Jones, D. A. (2002). Effects of resistance exercise volume and nutritional supplementation on anabolic and catabolic hormones. European Journal of Applied Physiology , 86, 315-321. 
Wright, D. A., Sherman, W. M., \& Dernbach, A. R. (1991). Carbohydrate feedings before, during, or in combination improve cycling endurance performance. Journal of Applied Physiology , 71 (3), 1082-1088.

Yaspelkis, B. B., Patterson, J. G., Anderla, P. A., Ding, Z., \& Ivy, J. L. (1993). Carbohydrate supplementation spares muscle glycogen during variable-intensity exercise. Journal of Applied Physiology , 75 (4), 1477-1485.

Zawadzki, K. M., Yaspelkis, B. B., \& Ivy, J. L. (Journal of Applied Physiology). Carbohydrate-protein supplement increases the rate of muscle glycogen storage post exercise. 72, 1854-1859. 